\title{
Bile acid excess impairs thermogenic function in brown adipose tissue
}

Weinan Zhou ${ }^{1 *}$, Philip VanDuyne ${ }^{1 *}$, Chi Zhang ${ }^{2}$, Ryan Riessen ${ }^{1}$, Maribel Barragan $^{3}$, Blair M.

Rowitz $^{3,4,5}$, Margarita Teran-Garcia ${ }^{3,5,6}$, Stephen A. Boppart 2, 5, 7, 8, and Sayeepriyadarshini Anakk ${ }^{1 \#}$

${ }^{1}$ Department of Molecular and Integrative Physiology, University of Illinois at UrbanaChampaign, Urbana, IL.

${ }^{2}$ Beckman Institute for Advanced Science and Technology, University of Illinois at UrbanaChampaign, Urbana, IL.

${ }^{3}$ Division of Nutritional Sciences, University of Illinois at Urbana-Champaign, Urbana, IL.

${ }^{4}$ Carle Foundation Hospital, Urbana, IL.

${ }^{5}$ Carle Illinois College of Medicine, University of Illinois at Urbana-Champaign, Urbana, IL.

${ }^{6}$ Department of Human Development and Family Studies, University of Illinois at UrbanaChampaign, Urbana, IL.

${ }^{7}$ Department of Bioengineering, University of Illinois at Urbana-Champaign, Urbana, IL.

${ }^{8}$ Department of Electrical and Computer Engineering, University of Illinois at Urbana-

Champaign, Urbana, IL.

* These authors contributed equally to this work.

\# Corresponding: anakk@illinois.edu (S.A.) 


\section{Highlights}

- Mouse model of BA overload exhibits adipose defects, which is partially restored by housing at thermoneutrality.

- $\quad$ BAs are present in detectable concentrations in both BAT and WAT.

- Adipocytes express genes responsible for de novo synthesis, conjugation and transport of BAs, and accumulate BAs.

- Pathological accumulation of BAs impairs mitochondrial function leading to thermogenic defect.

\section{SUMMARY}

Bile acids (BAs) not only facilitate fat digestion but also protect against obesity. Here, we show that a genetic mouse model for BA overload (Farnesoid X receptor; Small heterodimer double knockout (DKO)) exhibits mitochondrial dysfunction resulting in a thermogenic defect. By housing DKO mice at thermoneutrality, the poor mitochondrial function in brown fat protects them from diet-induced obesity. Compared to control, we find higher adipose BA levels with excess accumulation of taurocholic acid in the DKO mice. We report that the expression of genes responsible for BA de novo synthesis, conjugation and transporters and accumulation of BAs are present in both brown and white adipocytes. We determine that BA overload is sufficient to cause adipocyte mitochondrial dysfunction and induce the expression of cellular senescence genes in vitro. Taken together, we uncover that BA levels within the adipose tissue may modulate its overall function. 


\section{INTRODUCTION}

Adipose tissue is an important regulator of whole-body energy homeostasis. There are two major types of adipose tissues: white (WAT) and brown (BAT). WAT is primarily an energy storage depot, while the energy-burning BAT is responsible for non-shivering thermogenesis (Cohen and Spiegelman, 2016). Adipose tissue undergoes constant remodeling, however, during obesity, excessive expansion occurs resulting in pro-inflammatory milieu and metabolic syndrome (Rosen and Spiegelman, 2014).

Bile acids (BAs) are cholesterol metabolites that are primarily synthesized in the liver and released into the small intestine to facilitate fat digestion and absorption (Chiang, 2004). BAs are also signaling molecules that activate nuclear receptor Farnesoid $\mathrm{X}$ receptor (FXR) and membrane G protein-coupled receptor TGR5, to regulate metabolic homeostasis in the liver, intestine and adipose tissue (Chavez-Talavera et al., 2017; Li and Chiang, 2014; Yang et al., 2018). Earlier studies have shown that physiological levels of circulating BAs can promote heat production in the BAT to increase energy expenditure and prevent diet-induced obesity via the TGR5 signaling pathway (Broeders et al., 2015; Watanabe et al., 2006). However, the BA concentrations and their role within the adipose tissue has not been fully explored. We examined and found that the mouse model of BA overload displayed a thermogenic defect. We tested if the defective thermogenesis is due to BAs. Then, we examined the concentrations and composition of BAs in brown and white fat depots. We also investigated whether genes involved in BA synthesis and transport are expressed de novo in the adipose tissue, and if BAs are accumulated within adipocytes. Finally, we evaluated the mitochondrial function and cellular senescence of adipocytes in the presence and absence of BAs in vitro. Intriguingly, our results indicate that the defective thermogenesis subsequent to excess BAs may contribute towards protection against diet-induced obesity in mice. 


\section{RESULTS}

\section{DKO Mice Display Reduced Fat Accumulation and Impaired Brown Adipose}

\section{Mitochondrial Function}

Analysis of the genetic mouse model of nuclear receptors (Farnesoid X receptor (FXR), Small heterodimer partner(SHP)) double knockout (DKO) mice revealed elevated levels of hepatic (Table 1) and circulating BAs as we previously described (Anakk et al., 2011; Desai et al., 2017).These DKO mice show lower body weight and resistance to fatty liver disease (Akinrotimi et al., 2017). These mice also exhibit decreased brown and white fat mass (Figure 1A) and reduced adipocyte size (Figures $1 \mathrm{~B}, 1 \mathrm{C}$ and $\mathrm{S} 1 \mathrm{~A}$ ) compared to WT mice even under normal chow diet. This finding correlated well with reduced expression of lipogenic genes in the BAT (C/Ebpa, Srebp1c) and WAT (C/Ebpa, Fasn) (Figure S1B), suggesting reduced lipid synthesis in DKO adipose tissue. Although, DKO WAT displayed increased mRNA levels of SCD1 (Figure S1B), a rate-limiting enzyme for the synthesis of unsaturated fatty acids (Lodhi et al., 2011), no alteration was seen in the degrees of unsaturation in both WAT and BAT compared to WT mice (Figure S1D).

Since BAT mitochondrial thermogenesis can promote fat burning (Cohen and Spiegelman, 2016), we examined mitochondrial function in the BAT and continually monitored the body temperature of DKO and WT mice. Surprisingly, DKO mice exhibited decreased expression of thermogenic genes Prdm16 and Ucp1, while other mitochondrial genes Pgc1a and Dio2 were unchanged (Figure 1D). Despite an increase in respiratory chain complex I activity, we determined significantly reduced activities of citrate synthase as well as respiratory chain complex III and IV (Figure 1E). Further, DKO mice exhibited lower body temperature than WT mice during the daytime but not the night (Figure 1F). WAT, on the other hand, displayed comparable levels of mitochondrial genes between DKO and WT mice when housed at RT (Figure S2A). These results suggest that DKO mice have decreased lipid biosynthesis, poor mitochondrial function and impaired heat production in the brown fat. 


\section{Thermoneutral Housing Negates Brown Fat Defect and Restore Weight Gain upon High- fat Diet Condition in the DKO mice}

To confirm impaired brown adipose mitochondrial function, we housed both WT and DKO mice at thermoneutrality $\left(\mathrm{TN}, 30^{\circ} \mathrm{C}\right)$, which excludes the BAT thermogenic effect (Boutant et al., 2017). TN reduced the expression of crucial thermogenic genes, Dio2 and Ucp1, in the BAT from both WT and DKO mice (Figure S2B). However, TN neither altered mRNA levels of thermogenic transcription factors Prdm16 and Pgc1a in the BAT nor WAT mitochondrial genes including Prdm16, Pgc1a, Dio2 and Cpt1a in WT mice (Figures S2A and S2B). Despite a slightly smaller adipocyte size, the brown fat mass was comparable between DKO and WT BAT (Figures 2A-2C and S3A). Similarly, the transcript profile of lipogenic genes C/Ebpa and Srebp1c between DKO and WT mice housed at $30^{\circ} \mathrm{C}$ was not different (Figure S3B). However, TN did not alter the reduction in white fat mass (Figure 2A) and adipocyte size (Figures 2B-2C and S3A), or the induction in WAT lipogenic gene Scd1 expression (Figure S3B) in DKO mice. These results indicate poor brown fat function may protect the DKO mice against obesity. If this is true, then the DKO mice housed at $30{ }^{\circ} \mathrm{C}$ will lose their protection and gain weight.

We then challenged DKO and WT mice with a 45\% high-fat diet (HFD) for 8 weeks at either RT or TN conditions. DKO mice gained a similar percentage in body weight as WT (Figure 3A) in response to HFD at TN and showed increased BAT mass but not in WAT mass (Figure 3B). Although at RT, the adipocytes were smaller in both brown and white fat from DKO than WT mice upon HFD (Figures 3C, 3D and S4A), TN housing led to an increase in BAT adipocyte size in both WT and DKO mice (Figures 3C, 3D and S4A). Housing did not alter the WAT adipocyte size (Figures 3C, 3D and S4A). Similarly, WAT gene expression profile showed that lipogenic genes were comparable between DKO and WT mice at RT (Figure S4B).

However, WT BAT at TN post-HFD revealed significant induction of Srebp1c while Pparg, Fasn and Scd1 were suppressed with no change in C/Ebpa transcript levels (Figure S4C). Despite lower expression of many of these genes including C/Ebpa levels at RT, DKO BAT was able to 
upregulate $\mathrm{C} /$ Ebpa as well as Srebp1c transcript levels to that of WT at $30^{\circ} \mathrm{C}$ (Figure S4C), while Pparg, Fasn and Scd1 were maintained at the reduced levels (Figure S4C). These results indicate that fat accumulation in the BAT of DKO mice under TN correlates to increasing C/EBPa and Srebp1c levels.

Similar to the findings upon chow, HFD-fed DKO mice also showed lower expression of thermogenic genes Prdm16, Pgc1a, Dio2 and Ucp1 in the BAT compared to HFD fed WT mice when housed at RT (Figure 3E). Moreover, levels of BAT thermogenic genes including Prdm16, Pgc1a and Ucp1 were suppressed in WT mice upon housing at $30^{\circ} \mathrm{C}$ to that of DKO BAT at TN (Figure 3E). On the other hand, housing conditions modestly altered WAT gene expression pattern except for Prdm16 expression, which was higher in the DKO mice only at RT. Pgc1a was induced, while Dio2 and Cpt1a levels were reduced in DKO compared to WT WAT at both housing conditions after HFD (Figure S5). Next, we examined whether the compromised function is accompanied with abnormal structure of mitochondria. Under $30^{\circ} \mathrm{C}$ housing, the total number of mitochondria including the ones with abnormal structural features increased, whereas mitochondria with the normal structure were reduced compared to the mice housed at RT (Figures 4A-4C). These structural changes were seen in both the genotypes. But, HFD fed DKO mice housed at TN displayed an exaggerated mitochondrial change compared to HFD fed WT mice. The ratio of normal mitochondria in the DKO BAT dramatically dropped with the majority of them revealing abnormal ultrastructure including irregular shape, loss of cristae or presence of myelin figures (Figures 4A and 4C), an indicator of mitochondrial damage or degeneration (Le Beux et al., 1969; Siskova et al., 2010).

\section{BAs are Present in the Adipose Tissue and Their Composition is Altered in the DKO Mice}

BAs have been shown to regulate lipid metabolism (Chavez-Talavera et al., 2017; Li and Chiang, 2014; Yang et al., 2018), so we investigated the local levels of BAs in the fat depots. Previous studies have reported the presence of BAs in the white fat of humans (Jantti et al., 
2014) and mice (La Frano et al., 2017). We analyzed and found detectable levels of BAs in both BAT and WAT. BA concentrations (Figure 5A and Table 2), composition (Figure 5B) and hydrophobicity indices (Figure 5C) were comparable between the two adipose depots of WT mice. However, DKO mice displayed higher concentrations of BAs in both depots $(\sim 16 \mathrm{x}$ more in the BAT and $\sim 6 \mathrm{x}$ in the WAT) and also revealed composition differences compared to the WT mice (Figures 5A and 5B). Both adipose depots in the DKO mice showed about a 3-fold increase in taurocholic acid (TCA) with a concomitant decrease in cholic acid (CA) indicating increased BA-conjugation. We also found an increase in $\beta$-muricholic acid ( $\beta-M C A)$ in the brown

fat of DKO mice. Intriguingly, DKO BAT showed reductions in secondary BAs that are generated by dehydroxylation or epimerization by the gut microbiota. For instance, deoxycholic acid (DCA) and tauro- $\omega$-muricholic acid (T- $\omega$-MCA) were decreased compared to WT mice. Similarly, DKO WAT also showed a robust decrease in secondary BA, DCA, but pronounced increases in primary BA, tauro- $\alpha-$ muricholic acid (T- $\alpha-M C A)$ (Figure 5B). Albeit high concentrations in the BAT and WAT, the composition changes result in a decrease in BA hydrophobicity in DKO compared to WT mice (Figure 5C).

\section{Adipocytes Express Genes Regulating the Synthesis, Conjugation and Transport of BAs and Accumulate BAs}

The elevated concentrations of primary BAs in the adipose tissues raise the question if they can be synthesized or transported locally. To address this, we examined (i) the expression of genes controlling BAs synthesis and transport from the systemic circulation into cells within the adipose tissue, as well as (ii) BA accumulation within adipocytes. We found the expression of BA synthesis genes in both mouse and human adipose tissues (Figures $6 \mathrm{~A}$ and $6 \mathrm{~B}$ ). Brown fat of WT mice expressed higher levels of BA synthesis genes Cyp7a1, Cyp27a1 and Cyp2c70 but lower levels of Cyp7b1 compared to the white fat (Figure 6A). Human WAT also expressed these BA synthesis genes with CYP8B1 being the highest expressed (Figure 6B). To detect if 
primary adipocytes reveal the same expression pattern, we investigated pre- and postdifferentiated primary adipocytes derived from either brown or white adipose depots. Primary adipocytes from both depots displayed expression of all the BA synthesis genes with higher Cyp7b1 and Cyp8b1 levels in the preadipocytes derived from the WAT compared to BAT. Both BAT and WAT preadipocytes exhibited a minimal Cyp27a1 and no detectable Cyp2c70 expression which were dramatically induced post-differentiation in these cultured cells. Further, Cyp8b1 levels were upregulated in the differentiated brown adipocyte cultures while unaltered in the white adipocyte cultures. Cyp7b1 levels were reduced after differentiation in both white and brown adipocyte cultures, which matched with the tissue expression pattern. Cyp7a1 expression remained unaltered irrespective of the origin of the preadipocyte culture and its differentiated state (Figures 6C). Next, we examined and found induction of BA conjugation genes S/c27a5 and Baat as well as transporter genes S/co1a6, S/co1b2 and S/c51b in differentiated brown adipocyte cultures (Figures 6D and 6E). Conversely, in the adipocytes derived from white adipose, we found that the expression of genes regulating BA conjugation and transport including Baat, S/co1a6, S/co1b2, S/c51a and S/c51b remained lower than the brown adipocytes and were unaltered irrespective of their differentiation status (Figures 6D and 6E). Further, we found the accumulation of fluorescent BAs, choly-lysyl-fluorescein (CLF), within both brown and white differentiated primary adipocytes upon CLF treatment (Figure 7). These findings demonstrate that genes regulating BA synthesis, conjugation and transport are expressed in adipose depots and they exhibit distinct expression profile between brown and white adipocytes. Importantly, functional BA uptake may occur in adipocytes.

\section{High BA Levels Reduce Mitochondrial Membrane Potential and Induce Cellular Senescence Gene Expression in Adipocytes}

Based on our findings that DKO BAT exhibit mitochondrial defect and that they accumulate high BA concentrations, we examined the role of BAs in adipocyte mitochondrial function. We 
treated differentiated primary adipocytes derived from either BAT or WAT with pathological concentrations of chenodeoxycholic acid (CDCA). We observed that high doses of CDCA did not affect cell viability (Figure S6), but was sufficient to reduce mitochondrial membrane potential (Figures $8 \mathrm{~A}$ and $8 \mathrm{~B}$ ) in both brown and white adipocytes. Further, CDCA treatment significantly decreased the expression of thermogenic genes including Prdm16, Pgc1a, Dio2 and Ucp1 in the adipocytes derived from brown fat (Figures 8C). Except for Prdm16, Pgc1a, Dio2 and Cpt1a levels were reduced upon CDCA treatment in white adipocytes as well (Figure 8D). These results reveal that the accumulation of CDCA can dramatically reduce mitochondrial membrane potential and gene expression in adipocytes.

Aging is a risk factor for disease and death (Harman, 1991), and is associated with fat loss, mitochondrial dysfunction and impaired thermogenesis in the BAT (Florez-Duquet and McDonald, 1998) as well as increased BA levels (Lee et al., 2016). To explore whether high BAs accelerate the aging of adipose tissue, we examined cellular senescence, the critical process underlying aging, in response to CDCA treatment, and found that high levels of CDCA increased the expression of senescence genes including $p 19^{A r f}, p 21$ and II-6 in brown adipocytes (Figure 8E). CDCA treatment upregulated $/ /-6$ without affecting the levels of $p 16^{\text {Ink4a }}$, $p 19^{\text {Arf }}$ and $p 21$ in white adipocytes (Figure 8F). These findings demonstrate that excessive CDCA may induce brown adipocyte senescence.

\section{DISCUSSION}

Several studies have shown that BAs can alleviate diet-induced obesity through activating brown fat (Broeders et al., 2015; Watanabe et al., 2006). While BA role in the regulation of lipid metabolism has been studied (Chavez-Talavera et al., 2017; Li and Chiang, 2014; Yang et al., 2018), their de novo function within the adipocytes is poorly understood.

We used the global Fxr; Shp DKO mouse model for BA overload (Anakk et al., 2011; Desai et al., 2017) that shows resistance to diet-induced obesity (Akinrotimi et al., 2017). To 
explore the adipose function, we examined BA concentrations and composition in both brown and white fat and found them elevated in DKO compared to WT mice. The systemic circulation can contribute to adipose BA levels (Jantti et al., 2014), therefore, we tested BA uptake transporters and found S/co1a6 (Tian et al., 2015), S/co1b2 (Csanaky et al., 2011; Slijepcevic et al., 2017) and S/c51a/b (Suga et al., 2019) were expressed in the adipose tissue. Surprisingly, we visualized the accumulation of a conjugated-cholic acid (CA) analogue by adipocytes. Due to the hydrophilic property of conjugated-CA (Roda et al., 1990), these results suggest that BAs may be taken up by adipocytes using a transporter-medicated mechanism. On the other hand, BA conjugation genes were robustly expressed in adipocytes indicating the possibility of de novo taurine conjugation within the adipose. Importantly, DKO mice showed decreased BA hydrophobicity in the adipose but increased BA hydrophobicity in the serum (Anakk et al., 2011). In particular, the levels of hydrophilic BAs $\alpha / \beta-M C A$ significantly increased in the fat depots while only moderately upregulated in the serum of DKO compared to WT mice (Desai et al., 2017). These results imply that de novo synthesis may contribute to the hydrophilic BAs in the adipose tissue. Taken together, these data suggest that both BA de novo synthesis and transport may contribute to the differential adipose BA composition and concentrations in DKO mice. Further studies will be needed to characterize functional BA transporters and the de novo synthesis pathway. Our findings are consistent with a recent report that shows the presence of BAs in the BAT (Zhang et al., 2019). To overcome the caveat of whole-body knockout of Fxr and Shp, we performed in vitro studies using primary adipocyte cultures with intact FXR and SHP signaling to tease apart the BA effect. Previous studies have shown that Fxr or Shp deletion does not alter the basal BAT thermogenic gene expression profile (Park et al., 2011; Zhang et al., 2012).

Since we observed mitochondrial defects in the BAT of DKO mice, we investigated if high concentrations of BAs are detrimental and affect adipocyte mitochondrial function. In line with previous findings that physiological levels of BAs promote brown fat activity (Broeders et 
al., 2015; Watanabe et al., 2006), low concentrations of CDCA increase the mitochondrial membrane potential of brown adipocytes (Figure S7). However, when the BA concentration exceeds $100 \mu \mathrm{M}$ as seen in the DKO mice, it impairs mitochondrial function (Figure S7 and 8C). Due to their detergent properties, intracellular accumulation of BAs can increase mitochondrial membrane permeability leading to mitochondrial depolarization, which eventually triggers cell injury or death in hepatocytes (Rodrigues and Steer, 2000; Rolo et al., 2000; Sokol et al., 2005) and cardiomyocytes (Ferreira et al., 2005). Interestingly, despite weakening mitochondrial function, elevated doses of BAs did not cause any defects in adipocyte viability. Because of hydrophobic BA-induced cell injury and hydrophilic BA-induced cell protection (de Aguiar Vallim et al., 2013; Katagiri et al., 1992; Perez and Briz, 2009), the cytotoxicity of high hydrophobic BAs may be ameliorated by the altered BA composition. Thus, both elevated levels and altered composition of BAs may modulate adipose mitochondrial function. Importantly, we detected that the poor mitochondrial function in brown fat can partially protect the DKO mice against obesity. Previous studies have reported that adipose-specific mitochondrial dysregulation causes adipocyte energy deficiency and fat loss (Vernochet et al., 2014; Yang et al., 2016). Taken together, BA-induced adipocyte mitochondrial failure may result in reduced fat accumulation in the DKO mice.

We mapped this brown fat defect in the DKO mice to declined Ucp1 expression and aging. UCP1 is responsible for brown adipose thermogenesis (Fedorenko et al., 2012), such that $U_{c p} 1$ deficient mice display mitochondrial disruptions, defective thermoregulation, and are resistant to diet-induced obesity with reduced white fat mass when housed at room temperature (Enerback et al., 1997; Kazak et al., 2017; Liu et al., 2003). Similarly, aged mice also show downregulation of UCP1 levels, mitochondrial dysfunction, reductions in brown fat heat production, and fat loss (Florez-Duquet and McDonald, 1998; Zoico et al., 2019). DKO mice and BA-induced adipocyte cultures exhibit overlapping phenotypes with both Ucp1 knockout and 
aged mice (Table 3), suggesting that BA overload-induced mitochondrial dysfunction correlates to reducing Ucp1 levels and brown adipose defects during aging.

Overall, our findings uncover a novel role for elevated levels of BAs in regulating brown adipose mitochondrial function and the subsequent thermogenesis.

\section{STAR METHODS}

Detailed methods are provided in the online version of this paper and include the following:

- KEY RESOURCES TABLE

- RESOURCE AVAILABILITY

- Lead Contact

- Materials Availability

- Data and Code Availability

- EXPERIMENTAL MODEL AND SUBJECT DETAILS

- Human Adipose Tissue

- Animals

- METHOD DETAILS

○ Histology

○ Quantitative Real-Time PCR

- Analysis of the Degree of Lipid Unsaturation

- Mitochondrial Respiratory Enzyme Activity Assay

- Bile Acid Analysis

- Adipocyte Cell Culture

- Bile Acid Accumulation Assay

- Mitochondrial Membrane Potential Assay

- Cell Viability Assay

- QUANTIFICATION AND STATISTICAL ANALYSIS 


\section{ACKNOWLEDGEMENTS}

This work was supported by start-up funds from the University of Illinois at Urbana-Champaign (to SA) and R01 DK113080 from NIDDK (to SA). The authors are grateful to Ms. Oludemilade Akinrotimi for her assistance with obtaining transmission electron microscopy samples for analysis, Mr. Shawn D'Souza for qRT-PCR, and Dr. Lee-Jun Wong's laboratory at Baylor College of Medicine for mitochondrial complex analysis. We also thank Ms. Lou Ann Miller from the Biological Electron Microscopy core at Materials Research Laboratory for performing transmission electron microscopy as well as the NIH West Coast Metabolomics Center at the University of California, Davis for performing adipose tissue BA analysis. We want to acknowledge the Microscopy Suite at the Beckman Institute, the University of Illinois at UrbanaChampaign for the access to the Raman micro-spectrometer used in the study.

\section{AUTHOR CONTRIBUTIONS}

W.Z., P.V. and S.A. conceived and designed research; B.M.R., M.T.G. and M.B. supplied human adipose samples; W.Z., P.V., C.Z., R.R. and S.A. performed experiments; W.Z. analyzed data; W.Z. and S.A. interpreted data; W.Z. generated figures; W.Z. and S.A. drafted the manuscript; all authors were involved in editing and revising the manuscript, and had final approval of the submitted and published versions.

\section{DECLARATION OF INTERESTS}

The authors declare no competing interests. 


\section{STAR METHODS}

\section{KEY RESOURCES TABLE}

\begin{tabular}{|c|c|c|}
\hline REAGENT or RESOURCE & SOURCE & IDENTIFIER \\
\hline \multicolumn{3}{|c|}{ Chemicals, Peptides, and Recombinant Proteins } \\
\hline Teklad global $18 \%$ protein rodent diet & Envigo & Cat\# 2918 \\
\hline $45 \%$ kcal fat diet & Envigo & Cat\# TD.08811 \\
\hline $10 \%$ neutral-buffered formalin & VWR & Cat\# 89370-094 \\
\hline Paraffin & SPI Supplies & Cat\# 02847A-AB \\
\hline Hematoxylin & Thermo Scientific & Cat\# 72-11L \\
\hline Eosin & Thermo Scientific & Cat\# 22-050-198 \\
\hline TRIzol reagent & Invitrogen & Cat\# 15-596-018 \\
\hline DNase I & New England Biolabs & Cat\# M0303S \\
\hline DMEM & Gibco & Cat\# 11-965-092 \\
\hline HEPES & Fisher Bioreagents & Cat\# BP310-500 \\
\hline $\mathrm{NaCl}$ & Fisher Chemical & Cat\# S271-500 \\
\hline $\mathrm{KCl}$ & Fisher Chemical & Cat\# P217-500 \\
\hline $\mathrm{CaCl}_{2}$ & Fisher Chemical & Cat\# C79-500 \\
\hline Dextrose (D-Glucose) & Fisher Chemical & Cat\# D16-500 \\
\hline Bovine serum albumin (BSA) & VWR & Cat\# 97061-420 \\
\hline Collagenase type I & $\begin{array}{l}\text { Worthington Biochemical } \\
\text { Corporation }\end{array}$ & Cat\# LS004196 \\
\hline Fetal bovine serum (FBS) & Corning & Cat\# MT35010CV \\
\hline Glutamine & Gibco & Cat\# A2916801 \\
\hline Penicillin-Streptomycin & Gibco & Cat\# 15140122 \\
\hline Sodium ascorbate & Sigma-Aldrich & Cat\# A4034 \\
\hline 3-Isobutyl-1-methylxanthine (IBMX) & Sigma-Aldrich & Cat\# 15879 \\
\hline Dexamethasone & Sigma-Aldrich & Cat\# D4902 \\
\hline Insulin & Sigma-Aldrich & Cat\# 10516 \\
\hline Rosiglitazone & Sigma-Aldrich & Cat\# R2408 \\
\hline 3,3',5-Triiodo-I-thyronine (T3) & Sigma-Aldrich & Cat\# T2877 \\
\hline Tetramethylrhodamine ethyl ester (TMRE) & Cayman Chemical & Cat\# 21426 \\
\hline Phosphate-buffered saline (PBS) & Lonza & Cat\# BW17-517Q \\
\hline $\begin{array}{l}\text { Carbonyl cyaninde 4-(trifluoromethoxy) } \\
\text { phenylhydrazone (FCCP) }\end{array}$ & Sigma-Aldrich & Cat\# C2920 \\
\hline Calcein AM & Invitrogen & Cat\# C1430 \\
\hline \multicolumn{3}{|l|}{ Critical Commercial Assays } \\
\hline Maxima reverse transcriptase & Thermo Scientific & Cat\# FEREP0743 \\
\hline SYBR green master mix & Applied Biosystems & Cat\# A25778 \\
\hline \multicolumn{3}{|l|}{ Oligonucleotides } \\
\hline See Table S1 for primers used for qRT-PCR & This paper & $\mathrm{N} / \mathrm{A}$ \\
\hline \multicolumn{3}{|l|}{ Experimental Models: Organisms/Strains } \\
\hline Mouse: Fxr, Shp double knockout (DKO) & Anakk et al., 2011 & $\mathrm{~N} / \mathrm{A}$ \\
\hline Mouse: C57BL/6 & Jackson Laboratory & Cat\# 000664 \\
\hline \multicolumn{3}{|l|}{ Software and Algorithms } \\
\hline GraphPad Prism 8 & GraphPad & $\begin{array}{l}\text { GraphPad } \\
\text { Software, Inc }\end{array}$ \\
\hline Adiposoft-ImageJ & ImageJ & ImageJ.nih.gov \\
\hline
\end{tabular}




\section{RESOURCE AVAILABILITY}

\section{Lead Contact}

Further information and requests for resources and reagents should be directed to and will be

fulfilled by the Lead Contact, Dr. Sayeepriyadarshini Anakk (anakk@illinois.edu).

\section{Materials Availability}

This study did not generate new unique reagents.

\section{Data and Code Availability}

This study did not generate any unique datasets or code.

\section{EXPERIMENTAL MODEL AND SUBJECT DETAILS}

\section{Human Adipose Tissue}

Human perigastric white adipose tissue samples were obtained from tissue normally discarded from obese patients undergoing Roux-en-Y gastric bypass surgery (Robinson et al., 2019). All procedures were approved by the Institutional Review Board (IRB) at Carle Foundation Hospital and the University of Illinois at Urban-Champaign (IRB protocol \# 14092). Informed consent was obtained from the subjects and the privacy rights of the subjects were observed.

\section{Animals}

The generation of global Farnesoid X receptor (Fxr); Small heterodimer partner (Shp) double knockout (DKO) mice has been described (Anakk et al., 2011). Male DKO and C57BL/6 wildtype (WT) mice at 8- to 10-week-old were used. These mice were bred and maintained on a 12:12 light/dark cycle with ad libitum access to tap water and a normal chow diet in a climatecontrolled $\left(23^{\circ} \mathrm{C}\right)$ animal facility at the University of Illinois at Urbana-Champaign. At 8-10 weeks, mice were fed a normal chow or $45 \%$ high-fat diet for 8 weeks to mimic obesogenic conditions and housed either at room temperature $(\mathrm{RT})\left(23^{\circ} \mathrm{C}\right)$ or at thermoneutrality $(\mathrm{TN}, 30$ $\left.{ }^{\circ} \mathrm{C}\right)$ to blunt brown fat thermogenic activity. 
Mice were weighed weekly. After 8 weeks, a subset of the mice was used for monitoring core body temperature fluctuation using a Comprehensive Laboratory Animal Monitoring System (CLAMS). Briefly, animals were surgically implanted with a transmitter in the abdominal cavity and acclimated to the CLAMS cages. Fluctuations in the body temperature were recorded over the subsequent 24-hour period. The rest of mice were sacrificed at the end of the experimental regimen. Interscapular brown, inguinal and gonadal white adipose tissues were collected for primary preadipocyte culture and analysis of histology, gene expression, the degree of lipid unsaturation, mitochondrial respiratory enzyme activity, and bile acid levels. All experiments were performed following the National Institutes of Health guidelines for the care and use of laboratory animals, and all procedures were approved by the Institutional Animal Care and Use Committee at the University of Illinois at Urbana-Champaign.

\section{METHOD DETAILS}

\section{Histology}

Adipose tissues were fixed in $10 \%$ neutral-buffered formalin for 24 hours at $4{ }^{\circ} \mathrm{C}$ and processed. Formalin-fixed tissues were embedded using paraffin and cut on a microtome at $5 \mu \mathrm{m}$ thickness and mounted onto charged glass microscope slides. Adipose sections were deparaffinized and stained with hematoxylin \& eosin using standard histological protocol. Adipocyte size was quantified using Adiposoft-ImageJ software. Transmission electron microscopy (TEM) was performed as previously described (Nardi et al., 2019).

\section{Quantitative Real-Time PCR}

Total RNA was isolated from primary pre- and post-differentiated adipocytes as well as snapfrozen adipose tissues using TRIzol reagent. Upon DNase I treatment, RNA was reverse transcribed into cDNA using a Maxima reverse transcriptase kit. Quantitative real-time PCR (qRT-PCR) was performed with SYBR green master mix using Applied Biosystems QuantStudio

7 Flex Real-Time PCR System. To determine relative expression values, the $2^{-\Delta \Delta C t}$ method was 
used, where triplicate Ct values for each sample were averaged and subtracted from those derived from housekeeping gene 36B4. All primers used are listed in Table S1.

\section{Analysis of the Degree of Lipid Unsaturation}

Adipose tissues were fixed in $10 \%$ neutral-buffered formalin for 24 hours at $4{ }^{\circ} \mathrm{C}$. Raman spectra of the adipose tissue were acquired using a Horiba LabRAM HR Raman spectroscopy imaging system. We used a $532 \mathrm{~nm}$ laser with an average power of $80 \mathrm{~mW}$ for excitation. The spectral acquisition speed was $5 \mathrm{~s}$. A confocal configuration was applied using the spectrometer with a pinhole size of $200 \mu \mathrm{m}$. The Raman spectra background removal and intensity analysis were performed using Origin 2020. The relative degree of saturation level in formalin-fixed adipose tissues was measured using the Raman peak ratio $3006 / 1441 \mathrm{~cm}^{-1}$ (You et al., 2016).

\section{Mitochondrial Respiratory Enzyme Activity Assay}

Activities of mitochondrial respiratory enzymes (i.e. complex I (NADH: ubiquinone

oxidoreductase), complex III (ubiquinol-cytochrome c oxidoreductase), complex IV (cytochrome c oxidase)) were measured using a temperature-controlled spectrophotometer (Ultraspec 6300 pro, Biochrom Ltd.) as previously described (Brautbar et al., 2008). Citrate synthase was used as a marker for mitochondrial content. Enzyme activities are expressed as $\mathrm{nmol} / \mathrm{min} / \mathrm{mg}$ of protein.

\section{Bile Acid Analysis}

Adipose bile acid (BA) analysis was performed in the NIH West Coast Metabolomics Center at the University of California, Davis. Adipose BAs were extracted from interscapular brown and gonadal white adipose tissue samples $(4-4.75 \mathrm{mg}$ ) as previously described (La Frano et al., 2017). Six internal standards (GCA-d4, TCDCA-d4, CA-d6, GCDCA-d4, CDCA-d4, DCA-d4) were added. BA levels were quantified by ultra-high performance liquid chromatography chromatography-triple quadruple mass spectrometry (UHPLC-TQ-MS/MS) (Thermo Fisher Scientific). Data were processed with Metaboanalyst 4.0 (https://www.metaboanalyst.ca/MetaboAnalyst/home.xhtml) (Xia et al., 2009). The 
concentrations of individual BAs were summed to derive the levels of total, primary, secondary, unconjugated and taurine-conjugated BAs (Fu et al., 2012). BA hydrophobicity index was calculated as previously reported (Fu et al., 2012; Heuman, 1989).

\section{Adipocyte Cell Culture}

Interscapular brown and inguinal white preadipocytes were isolated and cultured as described previously (Cannon and Nedergaard, 2001; Gao et al., 2017). Briefly, male C57BL/6 WT mice at 3- to 4-week-old were euthanized by isoflurane inhalational anesthesia followed by cervical dislocation. The interscapular brown and inguinal white adipose depots were harvested and minced with scissors in DMEM. Tissue fragments were incubated with digestion buffer $\left(\left(d_{d d H_{2}} \mathrm{O}\right.\right.$ containing HEPES (100 mM), NaCl (123 mM), KCl (5 mM), $\mathrm{CaCl}_{2}(1.3 \mathrm{mM})$, glucose $(5 \mathrm{mM})$, bovine serum albumin (BSA) $(1.5 \% \mathrm{w} / \mathrm{v})$ and collagenase type I $(2 \mathrm{mg} / \mathrm{mL}))$, and shaken at 300 rpm at $37^{\circ} \mathrm{C}$ for 1 hour. The digested solution was then passed through a $100-\mu m$ cell strainer and placed on ice for $20 \mathrm{~min}$. The infranatant below the top mature adipocyte layer was collected and passed through a $70-\mu \mathrm{m}$ cell strainer, followed by centrifugation at $500 \mathrm{~g}$ for 10 min. The cell pellet was washed with DMEM, centrifuged, and then resuspended with preadipocyte culture medium (DMEM containing 10\% fetal bovine serum (FBS), glutamine (4mM), HEPES (10 mM), Penicillin-Streptomycin (100 U/mL) and sodium ascorbate $(25 \mu \mathrm{g} / \mathrm{mL}))$. Two days post-confluency (Day 0), the brown or white preadipocytes were stimulated with preadipocyte induction medium (preadipocyte culture medium containing 3-Isobutyl-1methylxanthine (IBMX) $(0.5 \mathrm{mM})$, dexamethasone $(1 \mu \mathrm{M})$, insulin $(1 \mu \mathrm{g} / \mathrm{mL})$ and rosiglitazone $(1$ $\mu \mathrm{M}$ ) in the presence or absence of 3,3',5-Triiodo-I-thyronine (T3) (1 nM)). Two days later (Day 2), the induction medium was changed to adipocyte maintain medium (preadipocyte culture medium containing insulin $(1 \mu \mathrm{g} / \mathrm{mL})$ with or without T3 $(1 \mathrm{nM}))$. The maintain medium was changed every two days, and full differentiation of brown and white adipocytes was achieved by Day 6 and 8, respectively.

\section{Bile Acid Accumulation Assay}


Post-differentiated adipocytes and Hep G2 cells (positive control) were treated with a fluorescein-labeled BA, choly-lysyl-fluorescein (CLF, $5 \mu \mathrm{M})$ for 24 hours at $37^{\circ} \mathrm{C}$, followed by nuclear counterstain with Hoechst 33342 for 20 min (Barber et al., 2015; Kostrubsky et al., 2003). Cell cultures were then washed three time with PBS and imaged using an EVOS fluorescent microscope.

\section{Mitochondrial Membrane Potential Assay}

Mitochondrial membrane potential in live cells was determined by measuring the accumulation of tetramethylrhodamine ethyl ester (TMRE) in active mitochondria. After 24 hours BA treatment, post-differentiated adipocytes were stained with TMRE $(250 \mathrm{nM})$ for $30 \mathrm{~min}$ at $37^{\circ} \mathrm{C}$, and then washed once with $0.2 \%$ BSA/PBS. The fluorescence of TMRE stained mitochondria was then measured using a microplate reader at $\mathrm{Ex} / \mathrm{Em}=544 / 590 \mathrm{~nm}$. As a positive control for depolarization, uncoupler carbonyl cyaninde 4-(trifluoromethoxy) phenylhydrazone (FCCP) (20 $\mu \mathrm{M})$ was applied to cells 10 min before TMRE labeling.

\section{Cell Viability Assay}

Cell viability was measured using a cell-permeant dye, calcein AM. After BA treatment for 24 hours, post-differentiated adipocytes were stained with calcein AM $(1 \mu \mathrm{M})$ for 30 min at $37^{\circ} \mathrm{C}$. The fluorescence in cell samples was then measured using a microplate reader at $\mathrm{Ex} / \mathrm{Em}=$ $485 / 538 \mathrm{~nm}$.

\section{QUANTIFICATION AND STATISTICAL ANALYSIS}

Data were expressed as means \pm SEM. Statistical analyses were performed using GraphPad Prism 8 software. Differences between two groups were analyzed using Student's $t$ test, and multiple group comparisons were analyzed using a one-way or two-way ANOVA with a Fisher's LSD post hoc test. $P<0.05$ was considered statistically significant. 


\section{References}

Akinrotimi, O., Riessen, R., VanDuyne, P., Park, J.E., Lee, Y.K., Wong, L.J., Zavacki, A.M., Schoonjans, K., and Anakk, S. (2017). Small heterodimer partner deletion prevents hepatic steatosis and when combined with farnesoid $X$ receptor loss protects against type 2 diabetes in mice. Hepatology 66, 1854-1865.

Anakk, S., Watanabe, M., Ochsner, S.A., McKenna, N.J., Finegold, M.J., and Moore, D.D. (2011). Combined deletion of Fxr and Shp in mice induces Cyp17a1 and results in juvenile onset cholestasis. J Clin Invest 121, 86-95.

Barber, J.A., Stahl, S.H., Summers, C., Barrett, G., Park, B.K., Foster, J.R., and Kenna, J.G. (2015). Quantification of Drug-Induced Inhibition of Canalicular Cholyl-I-Lysyl-Fluorescein Excretion From Hepatocytes by High Content Cell Imaging. Toxicol Sci 148, 48-59.

Boutant, M., Kulkarni, S.S., Joffraud, M., Ratajczak, J., Valera-Alberni, M., Combe, R., Zorzano, A., and Canto, C. (2017). Mfn2 is critical for brown adipose tissue thermogenic function. EMBO J 36, 1543-1558.

Brautbar, A., Wang, J., Abdenur, J.E., Chang, R.C., Thomas, J.A., Grebe, T.A., Lim, C., Weng, S.W., Graham, B.H., and Wong, L.J. (2008). The mitochondrial 13513G>A mutation is associated with Leigh disease phenotypes independent of complex I deficiency in muscle. Mol Genet Metab 94, 485-490.

Broeders, E.P., Nascimento, E.B., Havekes, B., Brans, B., Roumans, K.H., Tailleux, A., Schaart, G., Kouach, M., Charton, J., Deprez, B., et al. (2015). The Bile Acid Chenodeoxycholic Acid Increases Human Brown Adipose Tissue Activity. Cell Metab 22, 418-426.

Cannon, B., and Nedergaard, J. (2001). Cultures of adipose precursor cells from brown adipose tissue and of clonal brown-adipocyte-like cell lines. Methods Mol Biol 155, 213-224.

Chavez-Talavera, O., Tailleux, A., Lefebvre, P., and Staels, B. (2017). Bile Acid Control of Metabolism and Inflammation in Obesity, Type 2 Diabetes, Dyslipidemia, and Nonalcoholic Fatty Liver Disease. Gastroenterology 152, 1679-1694 e1673.

Chiang, J.Y. (2004). Regulation of bile acid synthesis: pathways, nuclear receptors, and mechanisms. J Hepatol 40, 539-551.

Cohen, P., and Spiegelman, B.M. (2016). Cell biology of fat storage. Mol Biol Cell 27, 25232527.

Csanaky, I.L., Lu, H., Zhang, Y., Ogura, K., Choudhuri, S., and Klaassen, C.D. (2011). Organic anion-transporting polypeptide $1 \mathrm{~b} 2$ (Oatp1b2) is important for the hepatic uptake of unconjugated bile acids: Studies in Oatp1b2-null mice. Hepatology 53, 272-281.

de Aguiar Vallim, T.Q., Tarling, E.J., and Edwards, P.A. (2013). Pleiotropic roles of bile acids in metabolism. Cell Metab 17, 657-669.

Desai, M.S., Mathur, B., Eblimit, Z., Vasquez, H., Taegtmeyer, H., Karpen, S.J., Penny, D.J., Moore, D.D., and Anakk, S. (2017). Bile acid excess induces cardiomyopathy and metabolic dysfunctions in the heart. Hepatology 65, 189-201.

Enerback, S., Jacobsson, A., Simpson, E.M., Guerra, C., Yamashita, H., Harper, M.E., and Kozak, L.P. (1997). Mice lacking mitochondrial uncoupling protein are cold-sensitive but not obese. Nature 387, 90-94.

Fedorenko, A., Lishko, P.V., and Kirichok, Y. (2012). Mechanism of fatty-acid-dependent UCP1 uncoupling in brown fat mitochondria. Cell 151, 400-413.

Ferreira, M., Coxito, P.M., Sardao, V.A., Palmeira, C.M., and Oliveira, P.J. (2005). Bile acids are toxic for isolated cardiac mitochondria: a possible cause for hepatic-derived cardiomyopathies? Cardiovasc Toxicol 5, 63-73.

Florez-Duquet, M., and McDonald, R.B. (1998). Cold-induced thermoregulation and biological aging. Physiol Rev 78, 339-358.

Fu, Z.D., Csanaky, I.L., and Klaassen, C.D. (2012). Gender-divergent profile of bile acid homeostasis during aging of mice. PLoS One 7, e32551. 
Gao, W., Kong, X., and Yang, Q. (2017). Isolation, Primary Culture, and Differentiation of Preadipocytes from Mouse Brown Adipose Tissue. Methods Mol Biol 1566, 3-8.

Harman, D. (1991). The aging process: major risk factor for disease and death. Proc Natl Acad Sci U S A 88, 5360-5363.

Heuman, D.M. (1989). Quantitative estimation of the hydrophilic-hydrophobic balance of mixed bile salt solutions. J Lipid Res 30, 719-730.

Jantti, S.E., Kivilompolo, M., Ohrnberg, L., Pietilainen, K.H., Nygren, H., Oresic, M., and Hyotylainen, T. (2014). Quantitative profiling of bile acids in blood, adipose tissue, intestine, and gall bladder samples using ultra high performance liquid chromatography-tandem mass spectrometry. Anal Bioanal Chem 406, 7799-7815.

Katagiri, K., Nakai, T., Hoshino, M., Hayakawa, T., Ohnishi, H., Okayama, Y., Yamada, T., Ohiwa, T., Miyaji, M., and Takeuchi, T. (1992). Tauro-beta-muricholate preserves choleresis and prevents taurocholate-induced cholestasis in colchicine-treated rat liver. Gastroenterology 102, 1660-1667.

Kazak, L., Chouchani, E.T., Stavrovskaya, I.G., Lu, G.Z., Jedrychowski, M.P., Egan, D.F., Kumari, M., Kong, X., Erickson, B.K., Szpyt, J., et al. (2017). UCP1 deficiency causes brown fat respiratory chain depletion and sensitizes mitochondria to calcium overload-induced dysfunction. Proc Natl Acad Sci U S A 114, 7981-7986.

Kirkland, J.L., and Dobson, D.E. (1997). Preadipocyte function and aging: links between agerelated changes in cell dynamics and altered fat tissue function. J Am Geriatr Soc 45, 959-967. Kirkland, J.L., Tchkonia, T., Pirtskhalava, T., Han, J., and Karagiannides, I. (2002). Adipogenesis and aging: does aging make fat go MAD? Exp Gerontol 37, 757-767. Kostrubsky, V.E., Strom, S.C., Hanson, J., Urda, E., Rose, K., Burliegh, J., Zocharski, P., Cai, H., Sinclair, J.F., and Sahi, J. (2003). Evaluation of hepatotoxic potential of drugs by inhibition of bile-acid transport in cultured primary human hepatocytes and intact rats. Toxicol Sci 76, 220228.

La Frano, M.R., Hernandez-Carretero, A., Weber, N., Borkowski, K., Pedersen, T.L., Osborn, O., and Newman, J.W. (2017). Diet-induced obesity and weight loss alter bile acid concentrations and bile acid-sensitive gene expression in insulin target tissues of C57BL/6J mice. Nutr Res 46, 11-21.

Le Beux, Y., Hetenyi, G., Jr., and Phillips, M.J. (1969). Mitochondrial myelin-like figures: a nonspecific reactive process of mitochondrial phospholipid membranes to several stimuli. $Z$ Zellforsch Mikrosk Anat 99, 491-506.

Lee, G., Lee, H., Hong, J., Lee, S.H., and Jung, B.H. (2016). Quantitative profiling of bile acids in rat bile using ultrahigh-performance liquid chromatography-orbitrap mass spectrometry: Alteration of the bile acid composition with aging. J Chromatogr B Analyt Technol Biomed Life Sci 1031, 37-49.

$\mathrm{Li}, \mathrm{T}$., and Chiang, J.Y. (2014). Bile acid signaling in metabolic disease and drug therapy. Pharmacol Rev 66, 948-983.

Liu, X., Rossmeisl, M., McClaine, J., Riachi, M., Harper, M.E., and Kozak, L.P. (2003).

Paradoxical resistance to diet-induced obesity in UCP1-deficient mice. J Clin Invest 111, 399407.

Lodhi, I.J., Wei, X., and Semenkovich, C.F. (2011). Lipoexpediency: de novo lipogenesis as a metabolic signal transmitter. Trends Endocrinol Metab 22, 1-8.

Mancuso, P., and Bouchard, B. (2019). The Impact of Aging on Adipose Function and Adipokine Synthesis. Front Endocrinol (Lausanne) 10, 137.

Nardi, J.B., Miller, L.A., and Bee, C.M. (2019). Interfaces between microbes and membranes of host epithelial cells in hemipteran midguts. J Morphol 280, 1046-1060.

Park, Y.J., Kim, S.C., Kim, J., Anakk, S., Lee, J.M., Tseng, H.T., Yechoor, V., Park, J., Choi, J.S., Jang, H.C., et al. (2011). Dissociation of diabetes and obesity in mice lacking orphan nuclear receptor small heterodimer partner. J Lipid Res 52, 2234-2244. 
Perez, M.J., and Briz, O. (2009). Bile-acid-induced cell injury and protection. World J Gastroenterol 15, 1677-1689.

Robinson, K.N., Rowitz, B., Oliphant, U.J., Donovan, S.M., and Teran-Garcia, M. (2019). Larger omental adipocytes correlate with greater Fetuin-A reduction following sleeve gastrectomy. BMC Obes 6, 15.

Roda, A., Minutello, A., Angellotti, M.A., and Fini, A. (1990). Bile acid structure-activity relationship: evaluation of bile acid lipophilicity using 1-octanol/water partition coefficient and reverse phase HPLC. J Lipid Res 31, 1433-1443.

Rodrigues, C.M., and Steer, C.J. (2000). Mitochondrial membrane perturbations in cholestasis. J Hepatol 32, 135-141.

Rolo, A.P., Oliveira, P.J., Moreno, A.J., and Palmeira, C.M. (2000). Bile acids affect liver mitochondrial bioenergetics: possible relevance for cholestasis therapy. Toxicol Sci 57, 177185.

Rosen, E.D., and Spiegelman, B.M. (2014). What we talk about when we talk about fat. Cell 156, 20-44.

Seidell, J.C., and Visscher, T.L. (2000). Body weight and weight change and their health implications for the elderly. Eur J Clin Nutr 54 Suppl 3, S33-39.

Siskova, Z., Mahad, D.J., Pudney, C., Campbell, G., Cadogan, M., Asuni, A., O'Connor, V., and Perry, V.H. (2010). Morphological and functional abnormalities in mitochondria associated with synaptic degeneration in prion disease. Am J Pathol 177, 1411-1421.

Slijepcevic, D., Roscam Abbing, R.L.P., Katafuchi, T., Blank, A., Donkers, J.M., van Hoppe, S., de Waart, D.R., Tolenaars, D., van der Meer, J.H.M., Wildenberg, M., et al. (2017). Hepatic uptake of conjugated bile acids is mediated by both sodium taurocholate cotransporting polypeptide and organic anion transporting polypeptides and modulated by intestinal sensing of plasma bile acid levels in mice. Hepatology 66, 1631-1643.

Sokol, R.J., Dahl, R., Devereaux, M.W., Yerushalmi, B., Kobak, G.E., and Gumpricht, E. (2005). Human hepatic mitochondria generate reactive oxygen species and undergo the permeability transition in response to hydrophobic bile acids. J Pediatr Gastroenterol Nutr 41, 235-243. St-Onge, M.P., and Gallagher, D. (2010). Body composition changes with aging: the cause or the result of alterations in metabolic rate and macronutrient oxidation? Nutrition 26, 152-155. Suga, T., Yamaguchi, H., Ogura, J., and Mano, N. (2019). Characterization of conjugated and unconjugated bile acid transport via human organic solute transporter alpha/beta. Biochim Biophys Acta Biomembr 1861, 1023-1029.

Tchkonia, T., Morbeck, D.E., Von Zglinicki, T., Van Deursen, J., Lustgarten, J., Scrable, H., Khosla, S., Jensen, M.D., and Kirkland, J.L. (2010). Fat tissue, aging, and cellular senescence. Aging Cell 9, 667-684.

Tian, J., Keller, M.P., Oler, A.T., Rabaglia, M.E., Schueler, K.L., Stapleton, D.S., Broman, A.T., Zhao, W., Kendziorski, C., Yandell, B.S., et al. (2015). Identification of the Bile Acid Transporter Slco1a6 as a Candidate Gene That Broadly Affects Gene Expression in Mouse Pancreatic Islets. Genetics 201, 1253-1262.

Vernochet, C., Damilano, F., Mourier, A., Bezy, O., Mori, M.A., Smyth, G., Rosenzweig, A., Larsson, N.G., and Kahn, C.R. (2014). Adipose tissue mitochondrial dysfunction triggers a lipodystrophic syndrome with insulin resistance, hepatosteatosis, and cardiovascular complications. FASEB J 28, 4408-4419.

Watanabe, M., Houten, S.M., Mataki, C., Christoffolete, M.A., Kim, B.W., Sato, H., Messaddeq, N., Harney, J.W., Ezaki, O., Kodama, T., et al. (2006). Bile acids induce energy expenditure by promoting intracellular thyroid hormone activation. Nature 439, 484-489.

Xia, J., Psychogios, N., Young, N., and Wishart, D.S. (2009). MetaboAnalyst: a web server for metabolomic data analysis and interpretation. Nucleic Acids Res 37, W652-660. 
Yang, H., Wu, J.W., Wang, S.P., Severi, I., Sartini, L., Frizzell, N., Cinti, S., Yang, G., and Mitchell, G.A. (2016). Adipose-Specific Deficiency of Fumarate Hydratase in Mice Protects Against Obesity, Hepatic Steatosis, and Insulin Resistance. Diabetes 65, 3396-3409.

Yang, Q., Vijayakumar, A., and Kahn, B.B. (2018). Metabolites as regulators of insulin sensitivity and metabolism. Nat Rev Mol Cell Biol 19, 654-672.

You, S., Tu, H., Zhao, Y., Liu, Y., Chaney, E.J., Marjanovic, M., and Boppart, S.A. (2016).

Raman Spectroscopic Analysis Reveals Abnormal Fatty Acid Composition in Tumor Micro- and Macroenvironments in Human Breast and Rat Mammary Cancer. Sci Rep 6, 32922.

Zhang, Y., Ge, X., Heemstra, L.A., Chen, W.D., Xu, J., Smith, J.L., Ma, H., Kasim, N., Edwards, P.A., and Novak, C.M. (2012). Loss of FXR protects against diet-induced obesity and accelerates liver carcinogenesis in ob/ob mice. Mol Endocrinol 26, 272-280.

Zhang, Y., Zheng, X., Huang, F., Zhao, A., Ge, K., Zhao, Q., and Jia, W. (2019).

Ursodeoxycholic Acid Alters Bile Acid and Fatty Acid Profiles in a Mouse Model of Diet-Induced Obesity. Front Pharmacol 10, 842.

Zoico, E., Rubele, S., De Caro, A., Nori, N., Mazzali, G., Fantin, F., Rossi, A., and Zamboni, M. (2019). Brown and Beige Adipose Tissue and Aging. Front Endocrinol (Lausanne) 10, 368. 


\section{Figure Legends}

Figure 1. DKO mice show decreased fat accumulation and BAT mitochondrial dysfunction upon normal chow diet

(A) Brown and white fat mass/body weight ratios of DKO and WT mice upon normal chow ( $n=8$ per group).

(B-C) Representative images (B) (scale bar: $200 \mu \mathrm{m}$ ) and quantification of adipocyte size (C) of H\&E-stained BAT ( $n=4-5$ mice per group) and WAT ( $n=6-7$ mice per group) sections of DKO and WT mice upon normal chow.

(D-E) Thermogenic gene expression (D) ( $n=7$ mice per group) and mitochondrial respiratory enzyme activity (E) ( $n=3$ mice per group) of the BAT from WT and DKO mice upon normal chow.

(F) Body temperature of DKO and WT mice for 24 hours upon normal chow ( $\mathrm{n}=7$ mice per group).

Data are represented as mean \pm SEM. ${ }^{*} P<0.05,{ }^{* *} P<0.01,{ }^{* *} P<0.001$ compared to WT mice.

Figure 2. Thermoneutrality abolishes DKO BAT defect in fat accumulation upon normal chow diet

(A) Brown and white fat mass/body weight ratios of DKO and WT mice upon normal chow housed at TN for 8 weeks ( $n=7-11$ per group).

(B-C) Representative images (B) (scale bar: $200 \mu \mathrm{m}$ ) and quantification of adipocyte size (C) of H\&E-stained BAT ( $n=7-11$ mice per group) and WAT ( $n=8-10$ mice per group) sections of DKO and WT mice upon normal chow housed at TN.

Data are represented as mean \pm SEM. ${ }^{*} P<0.05,{ }^{* * * *} P<0.0001$ compared to WT mice.

Figure 3. DKO mice display reduced fat accumulation upon HFD

(A-B) Body weight gain (A) ( $n=6-7$ mice per group) and fat mass/body weight ratios $(B)(n=7-8$ mice per group for RT, $n=5-6$ mice per group for TN) of DKO and WT mice housed at RT or TN upon HFD.

(C-D) Representative images (C) (scale bar: $200 \mu \mathrm{m}$ ) and quantification of adipocyte size (D) of H\&E-stained BAT ( $n=5-9$ mice per group for $R T$, $n=5-6$ mice per group for TN) and WAT ( $n=6$ mice per group for RT, $n=5$ mice per group for TN) sections from WT and DKO mice housed at RT or TN upon HFD. 
(E) Expression of thermogenic genes of BAT from DKO and WT mice housed at RT ( $n=6-8$ mice per group) or TN ( $n=5-6$ mice per group) upon HFD for 8 weeks.

Data are represented as mean \pm SEM. ${ }^{*} P<0.05,{ }^{* *} P<0.01,{ }^{* * *} P<0.001,{ }^{* * * *} P<0.0001$.

Figure 4. Thermoneutral housing alters BAT mitochondrial number and structure upon HFD

(A-C) Representative electron microscopy images (A) (abnormal mitochondria with irregular shape (asterisks), loss of cristae (empty arrowheads) or myelin figures (arrowheads), magnification: 10,000x) as well as quantification of mitochondria number (B) and structure (C) of BAT from DKO and WT mice housed at RT ( $n=3-4$ mice per group) or TN ( $n=3-5$ mice per group) upon HFD for 8 weeks.

Data are represented as mean \pm SEM. ${ }^{*} P<0.05,{ }^{* *} P<0.01,{ }^{* * * *} P<0.0001$.

Figure 5. DKO mice exhibit altered adipose BA composition

(A-C) Levels (A), composition (B) (BA percentage below $2 \%$ is not shown) and hydrophobicity

(C) of BAs in the BAT and WAT from DKO and WT mice upon normal chow ( $n=3$ mice per group).

Data are represented as mean \pm SEM. ${ }^{*} P<0.05,{ }^{* *} P<0.01$ compared to WT mice.

Figure 6. BA synthesis, conjugation and transporter genes are expressed in adipocytes (A-B) Expression of BA synthesis genes in the BAT and WAT from chow-fed WT mice (A) ( $n=6$ mice) and in the perigastric WAT from obese humans (B) ( $n=14$ individuals).

(C-E) Expression of BA synthesis (C), conjugation (D) and transporter (E) genes in primary preand post-differentiated adipocytes from BAT and WAT of chow-fed WT mice ( $n=3$ cultures). Data are represented as mean \pm SEM. ${ }^{*} P<0.05,{ }^{* \star} P<0.01,{ }^{* *} P<0.001,{ }^{* * * *} P<0.0001$.

Figure 7. BAs are accumulated within adipocytes

Representative images of accumulation of a fluorescein-labeled BA, CLF, within the differentiated brown and white adipocytes as well as Hep G2 liver cell line (positive control) upon CLF treatment for 24 hours (scale bar: $200 \mu \mathrm{m} ; \mathrm{n}=3$ cultures per group). Nuclei were stained with Hoechst 33342.

Figure 8. Pathological concentrations of CDCA impair adipocyte mitochondrial function and induce senescence gene expression 
(A-B) Representative images of TMRE staining of active mitochondria (A) (scale bar: $400 \mu \mathrm{m}$ ) and quantification of mitochondrial membrane potential $(B)$ of differentiated brown and white adipocytes upon CDCA treatment for 24 hours ( $n=4$ cultures per group).

(C-D) Expression of genes involved in thermogenesis in differentiated brown adipocytes (C) as well as genes regulating mitochondrial function in differentiated white adipocytes (D) in response to pathological concentrations of $C D C A$ ( $n=3$ cultures per group).

$(E-F)$ Expression of senescence marker genes in differentiated brown $(E)$ and white $(F)$ adipocytes in response to pathological concentrations of CDCA ( $n=3$ cultures per group).

Data are represented as mean \pm SEM. ${ }^{*} P<0.05,{ }^{* *} P<0.01,{ }^{* *} P<0.001,{ }^{* * *} P<0.0001$ compared to vehicle-treated adipocytes indicated by the dashed line. 
A Fat Mass

B
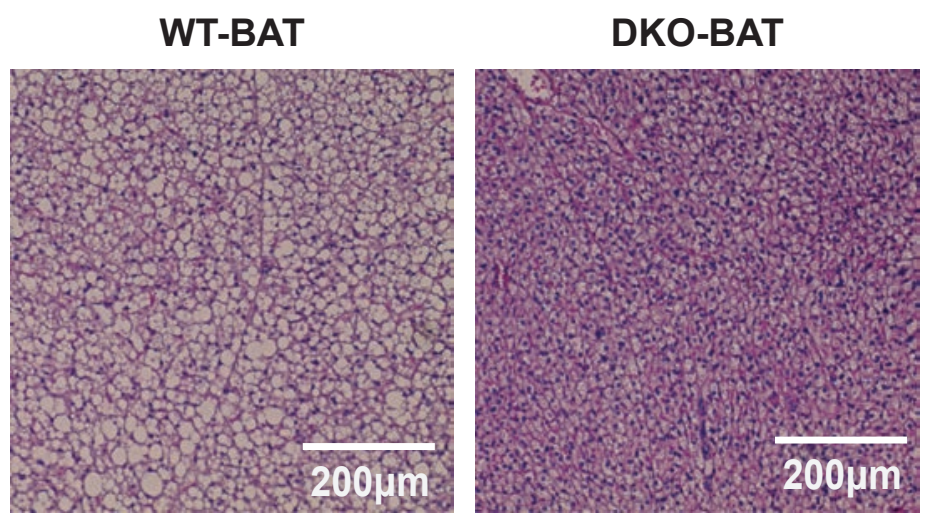

BAT Thermogenesis

D

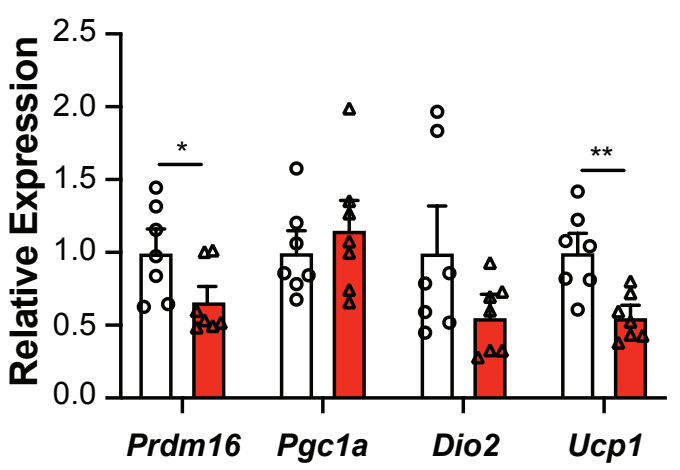

E

Adipose H\&E Stain

WT-WAT
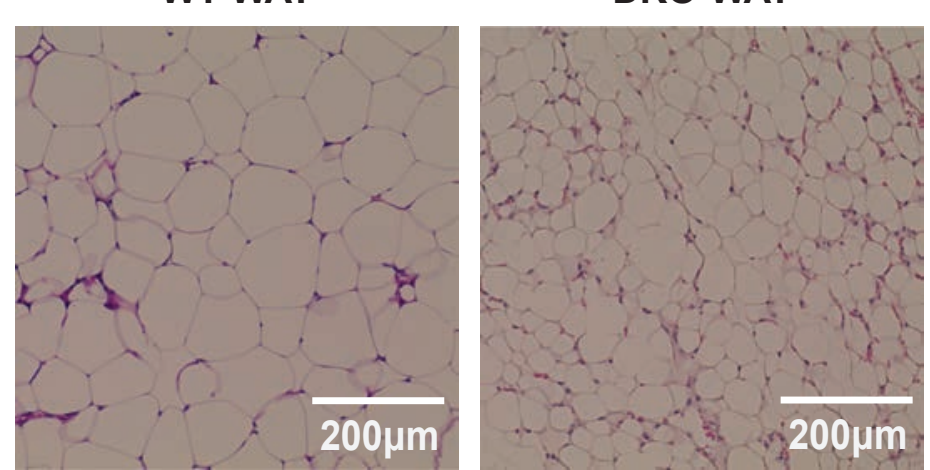

F

\section{Body Temperature} Respirtory Enzymes
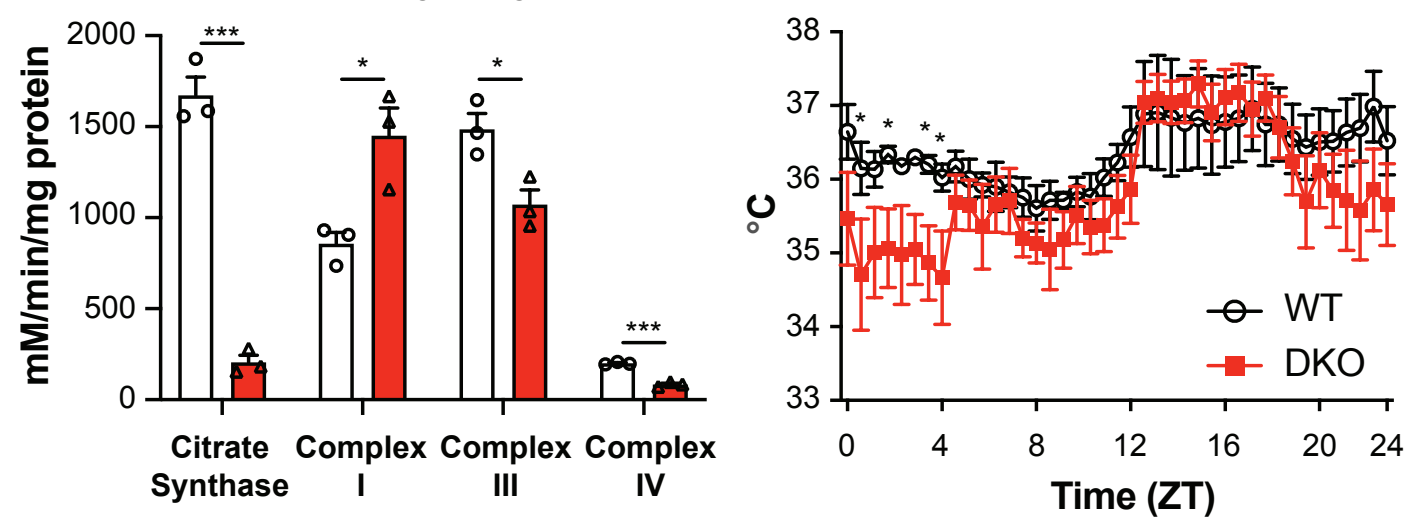

\section{Figure 1}


A

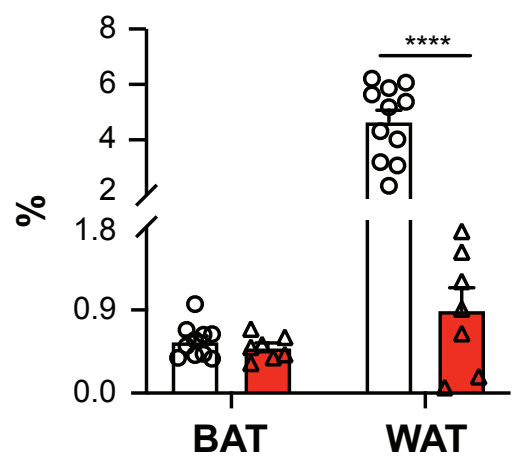

C Adipocyte Size at TN

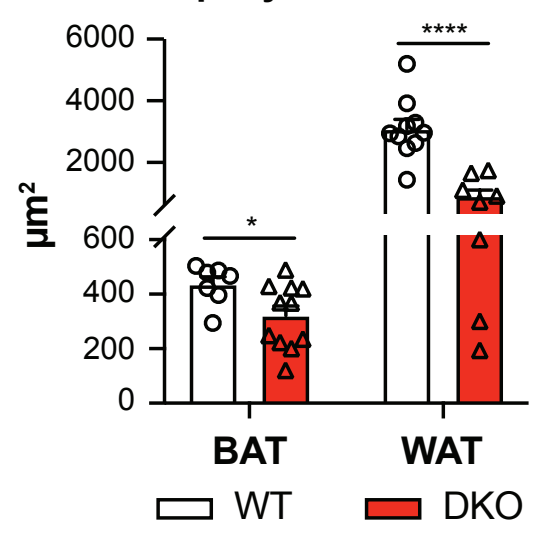

B H\&E Stain at TN
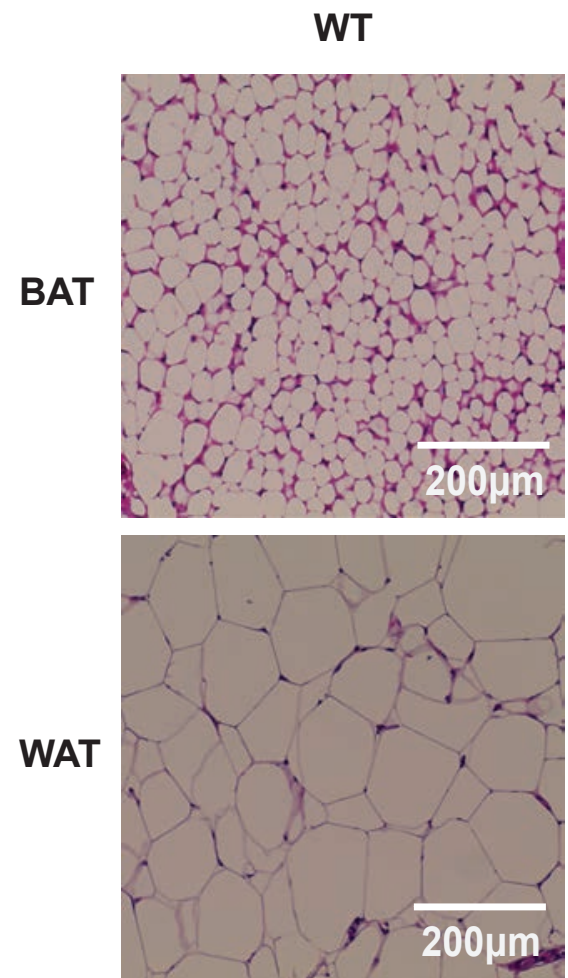

DKO
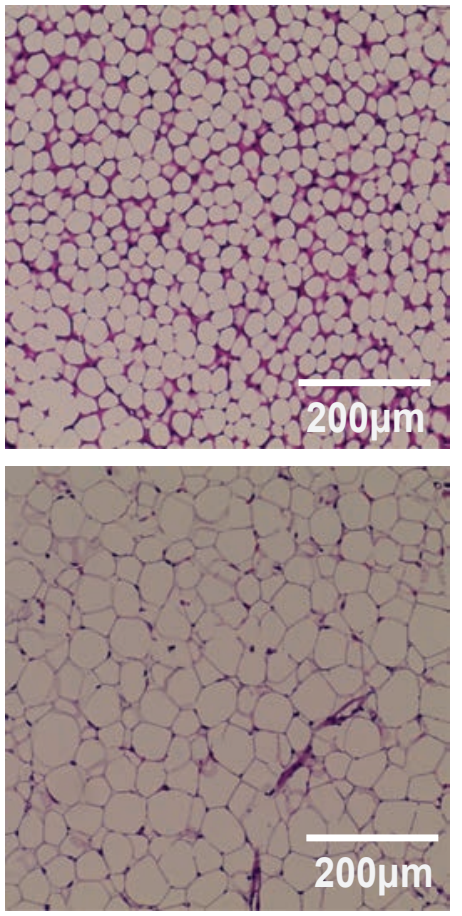

Figure 2 
A Weight Gain upon HFD at TN

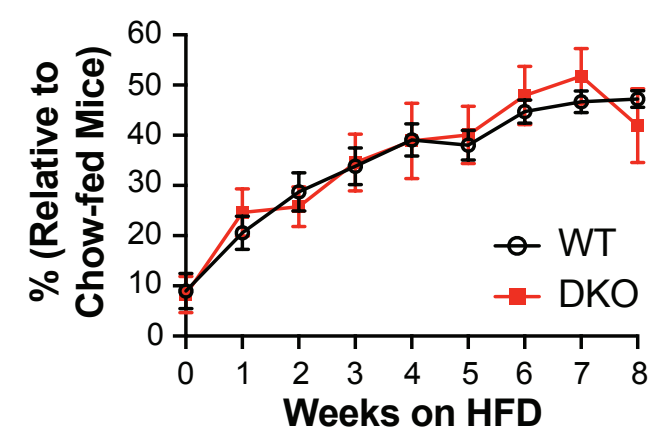

C

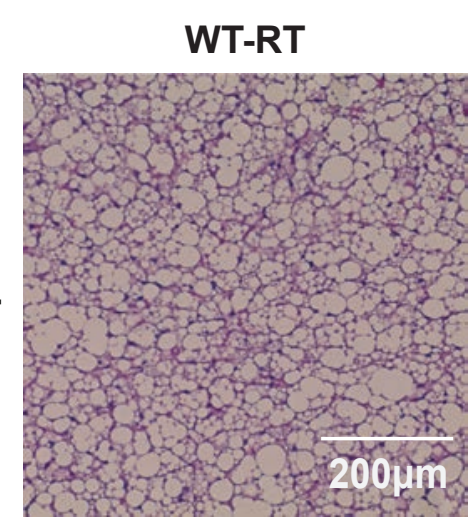

$200 \mu \mathrm{m}$

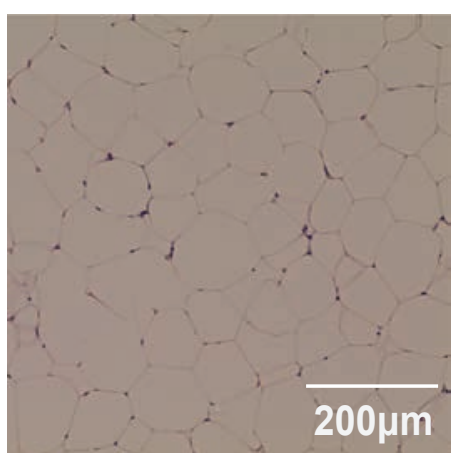

B BAT Mass upon HFD
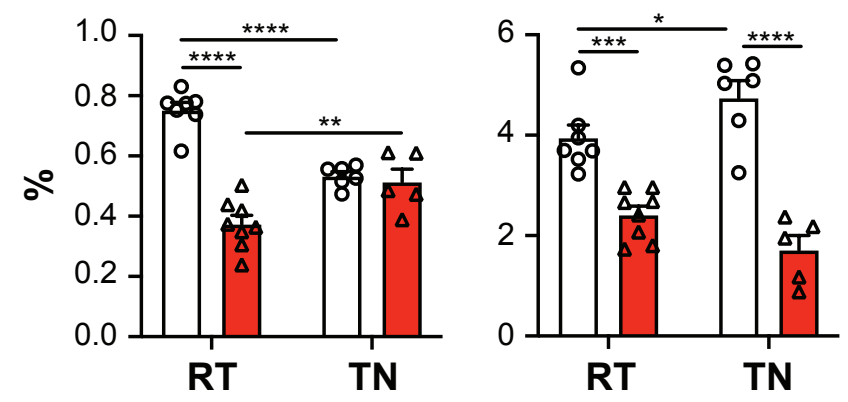

WAT Mass upon HFD
Adipose H\&E Stain upon HFD DKO-RT

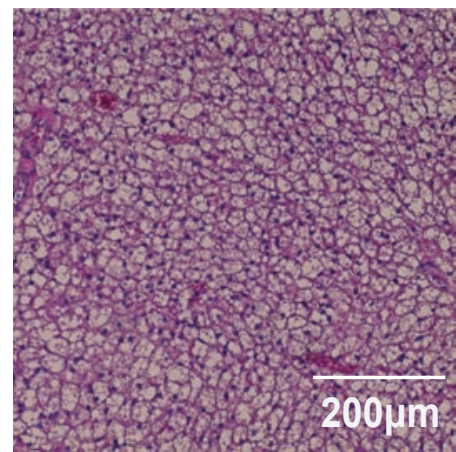

$200 \mu \mathrm{m}$
WT-TN
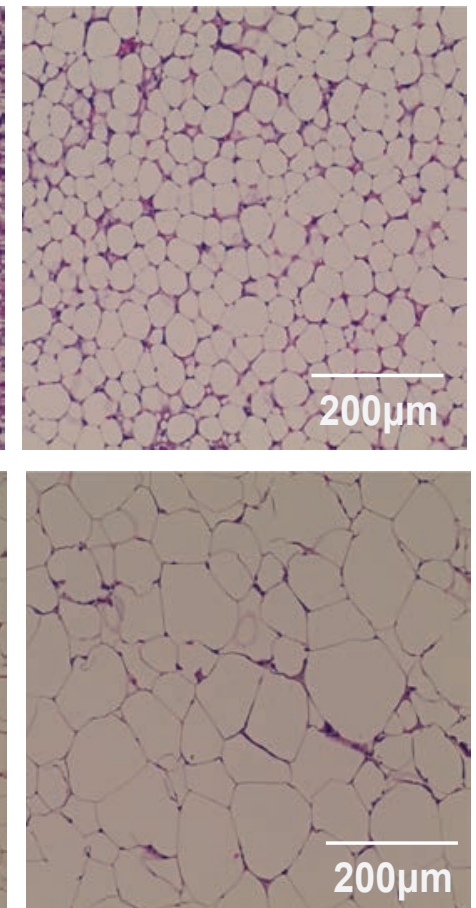

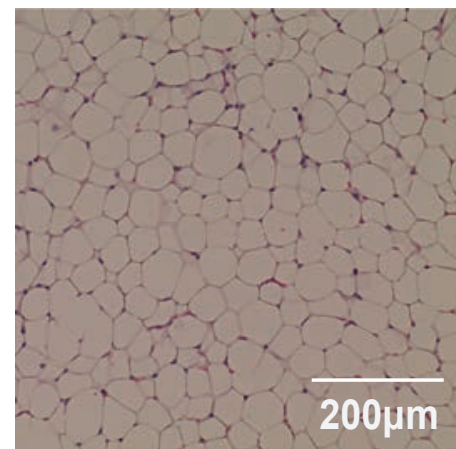

D Brown Adipocyte Size upon HFD

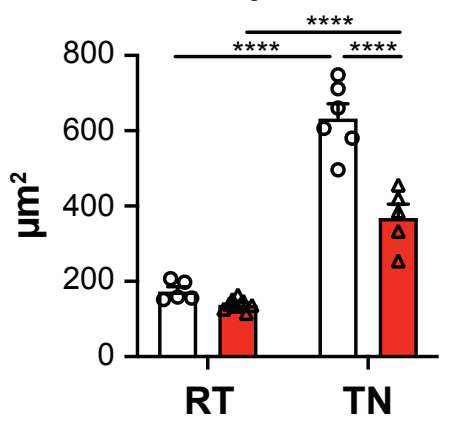

E
White Adipocyte

Size upon HFD

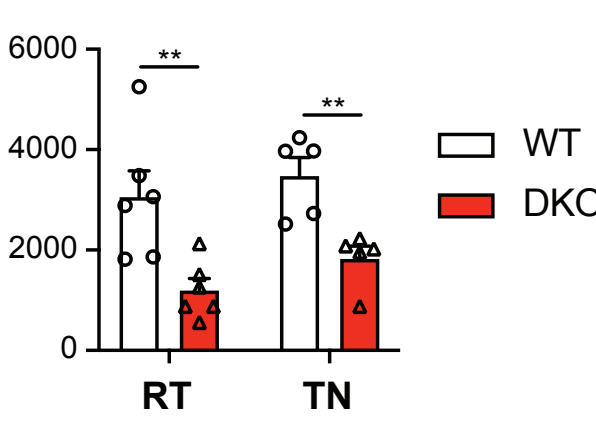

$200 \mu \mathrm{m}$
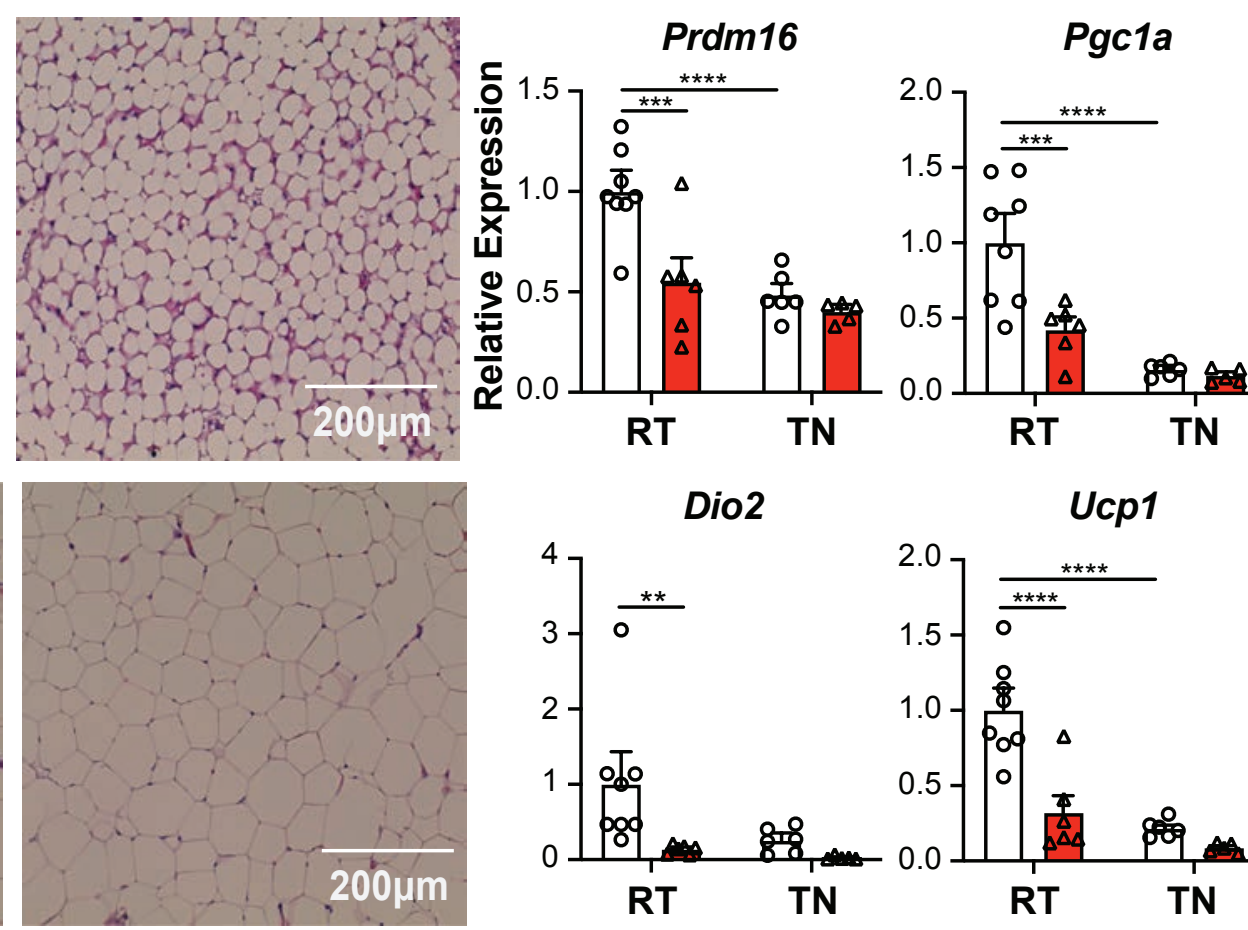

Figure 3 
A Brown Adipocyte Morphology upon HFD

WT-RT DKO-RT

WT-TN

DKO-TN
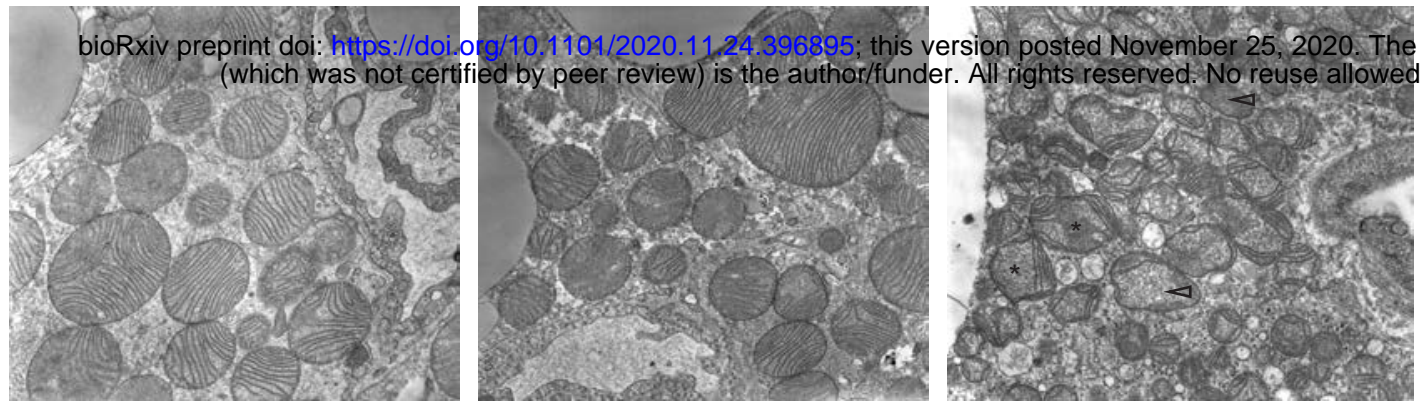

copyright holder for this preprint

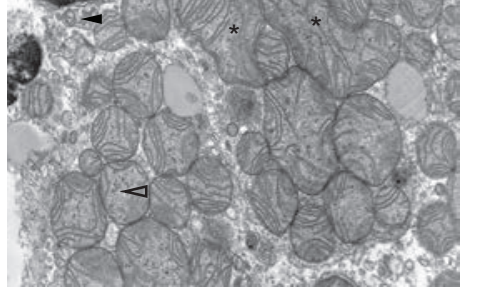

B Number of Mitochondria

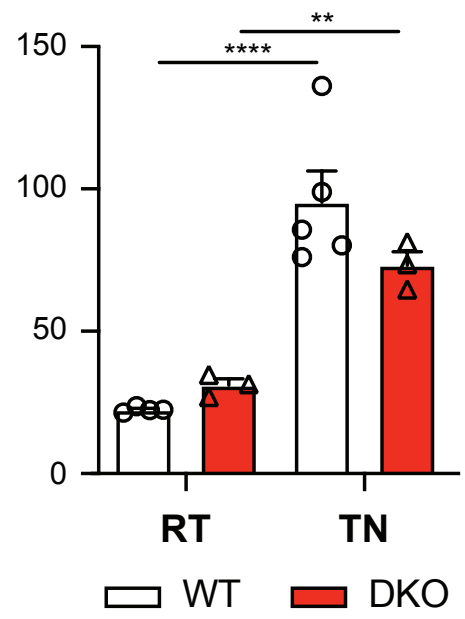

C Structure of Mitochondria

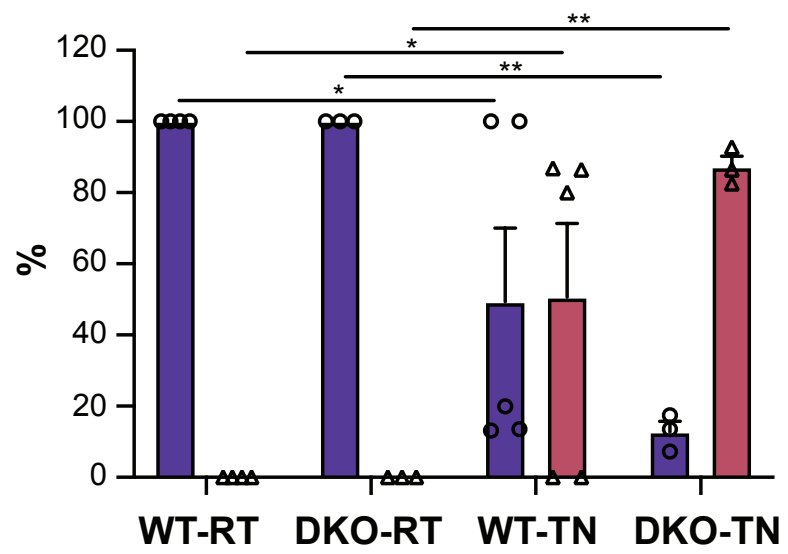

$\square$ Normal Mitochondria $\square$ Abnormal Mitochondria

Figure 4 


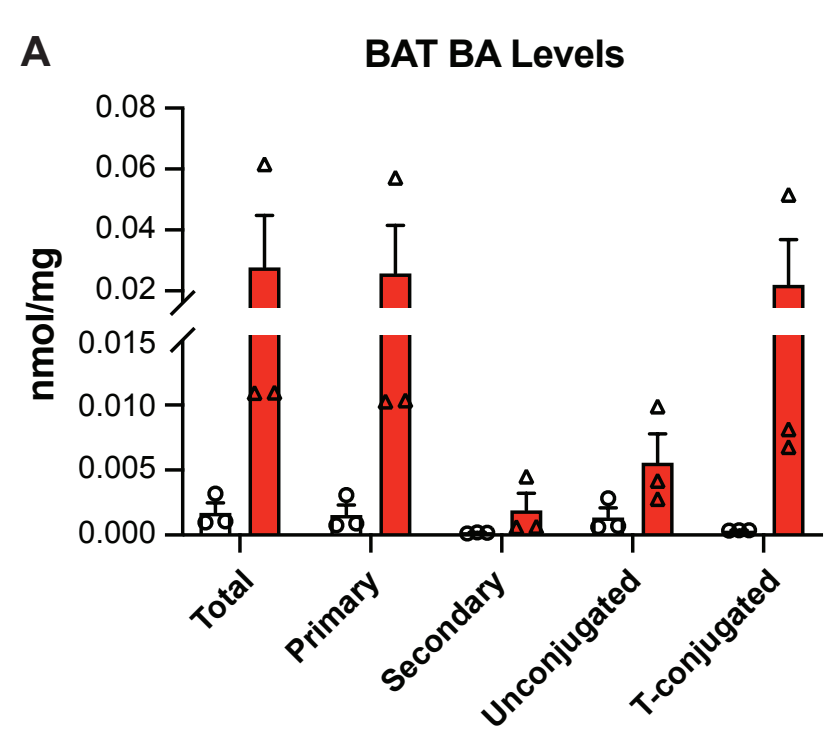

B

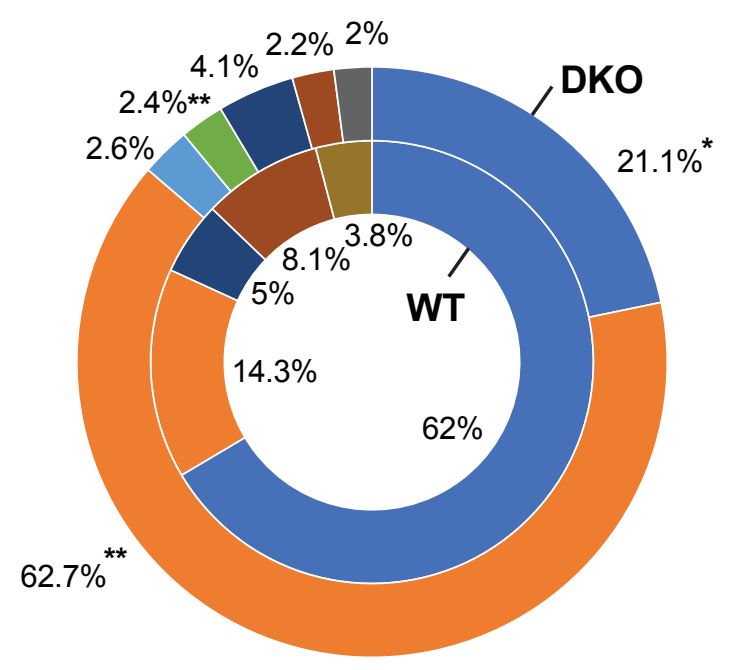

WAT BA Levels

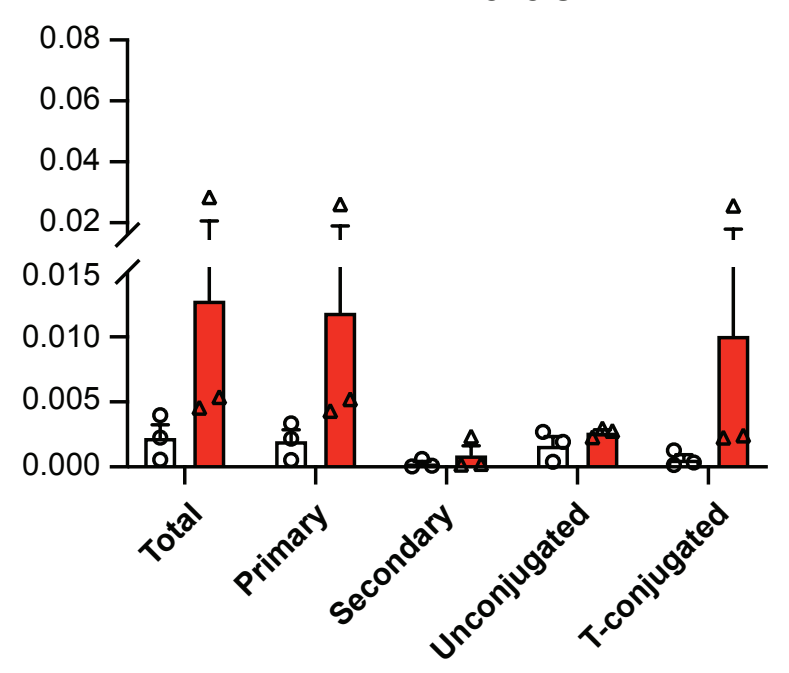

WAT BA Composition

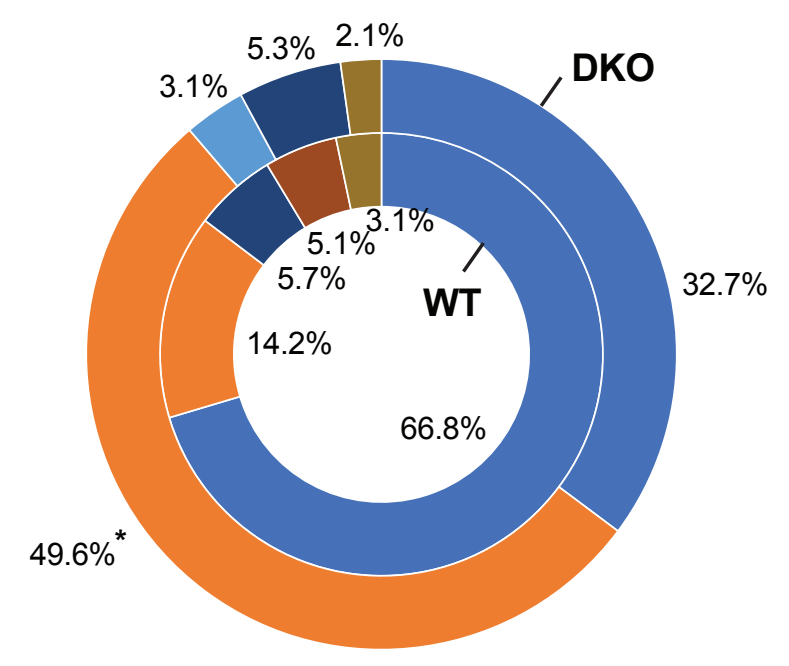

\section{BA Hydrophobicity Index}

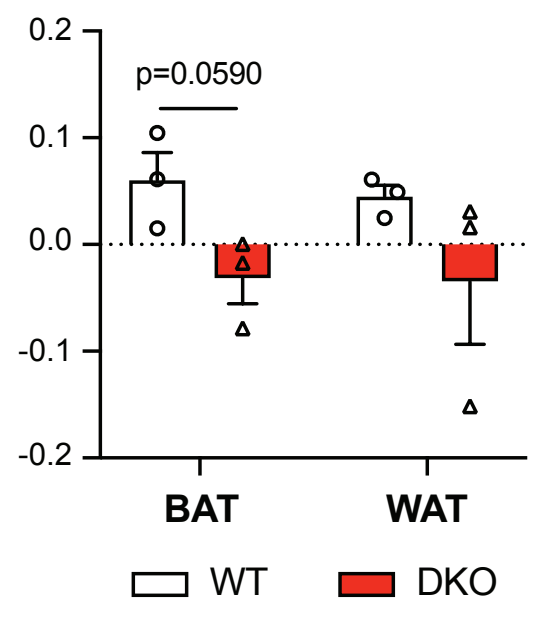

\section{Figure 5}


A

BA Synthesis

in Mouse Adipose Tissues
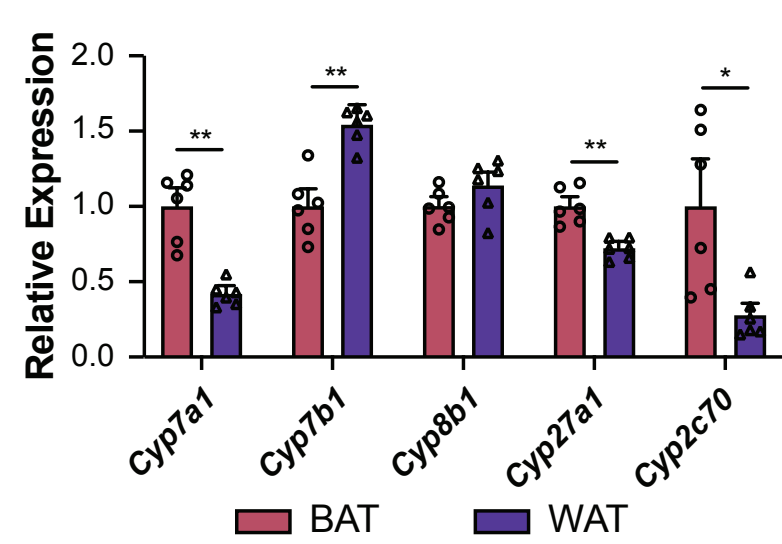

B BA Synthesis in Human WAT

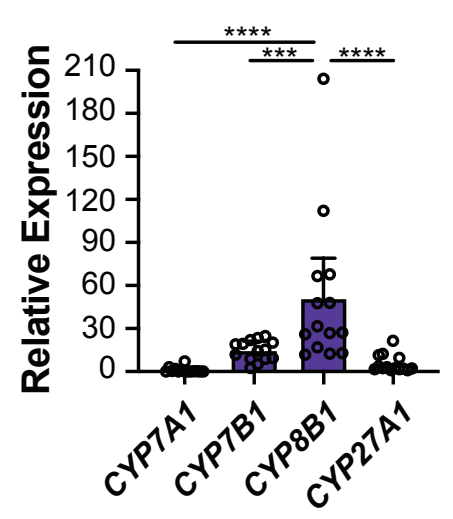

D BA Conjugation in Mouse Adipocytes

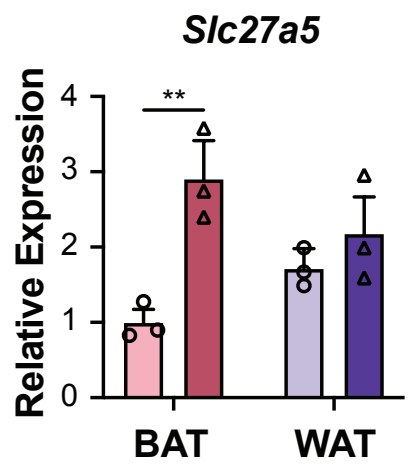

Baat

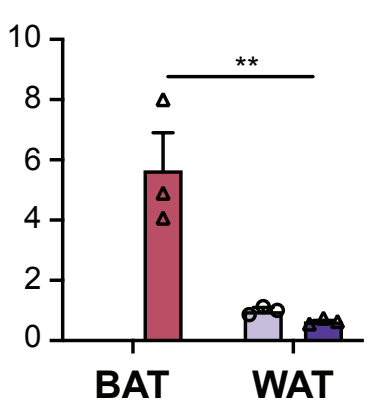

C

BA Synthesis in Mouse Adipocytes
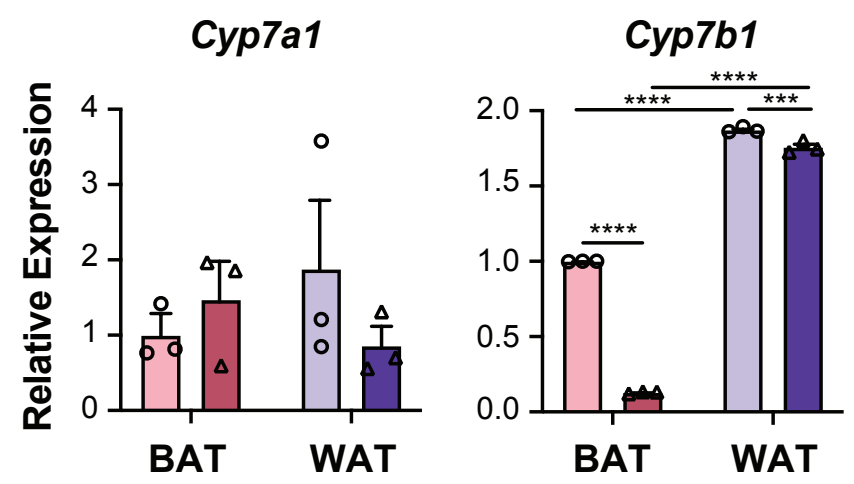

Cyp8b1

Cyp27a1

Cyp2c70
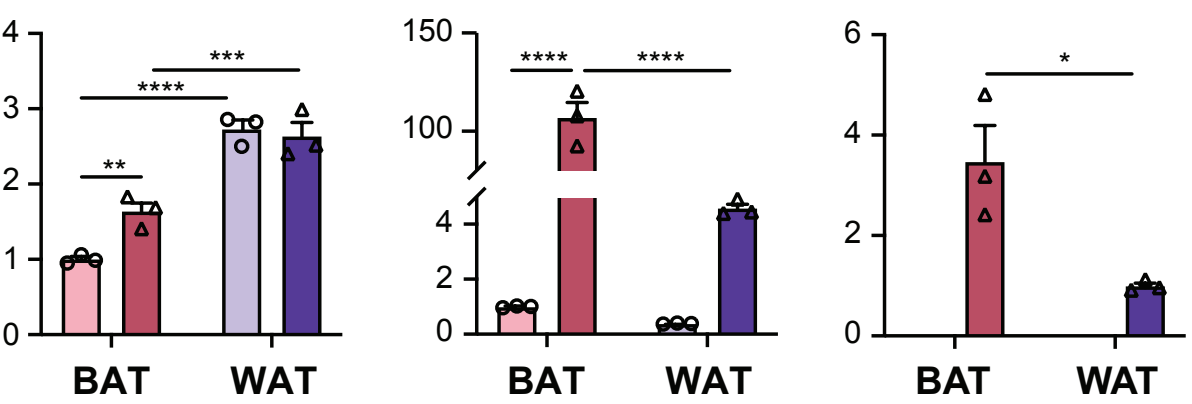

E

BA Transporter in Mouse Adipocytes

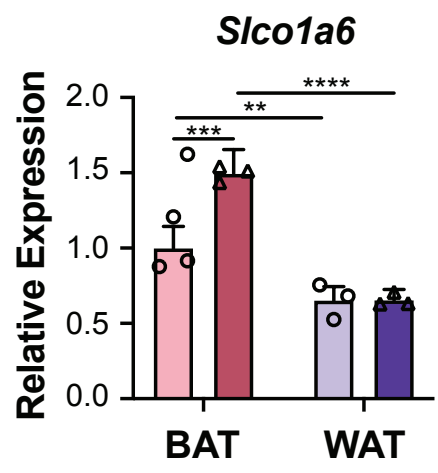
Slco1b2
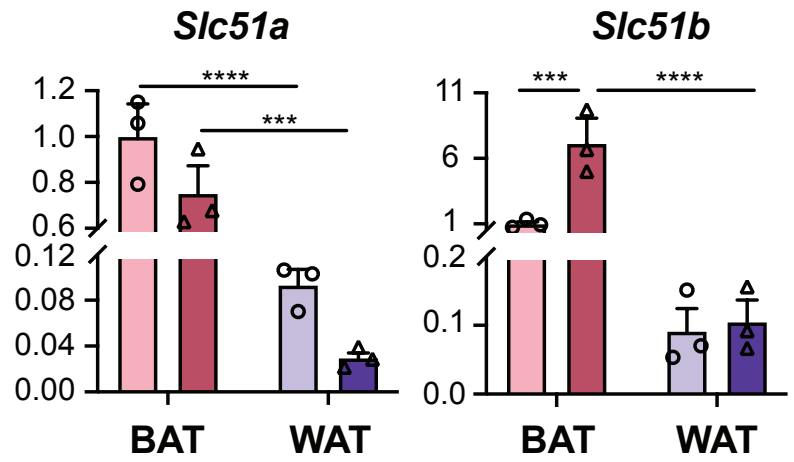

$\square$ Brown preadipocytes

$\square$ Differentiated brown adipocytes

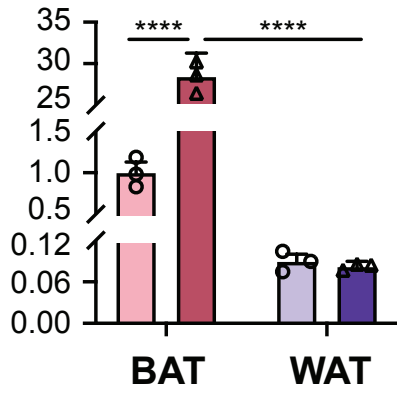

$$
\text { BAT WAT }
$$

\section{Figure 6}


Bright field

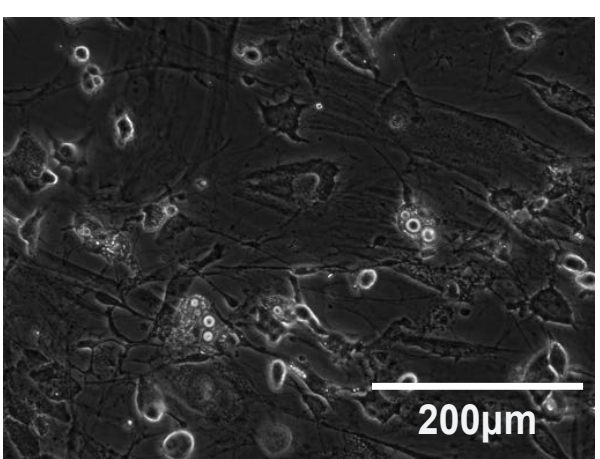

White

adipocytes

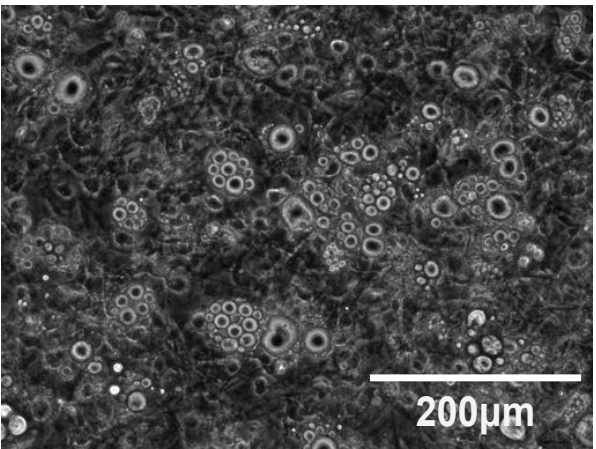

Hep G2

liver cells

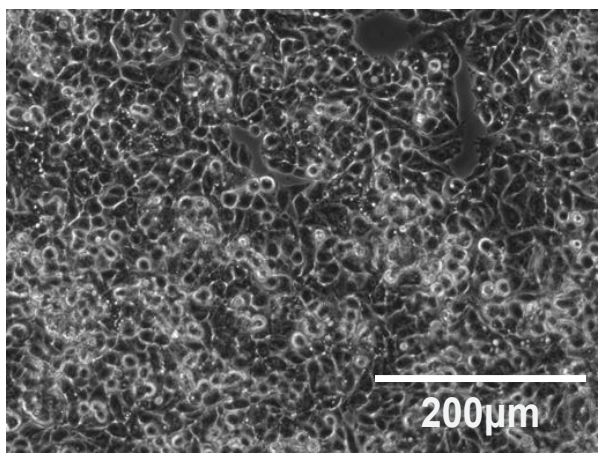

CLF
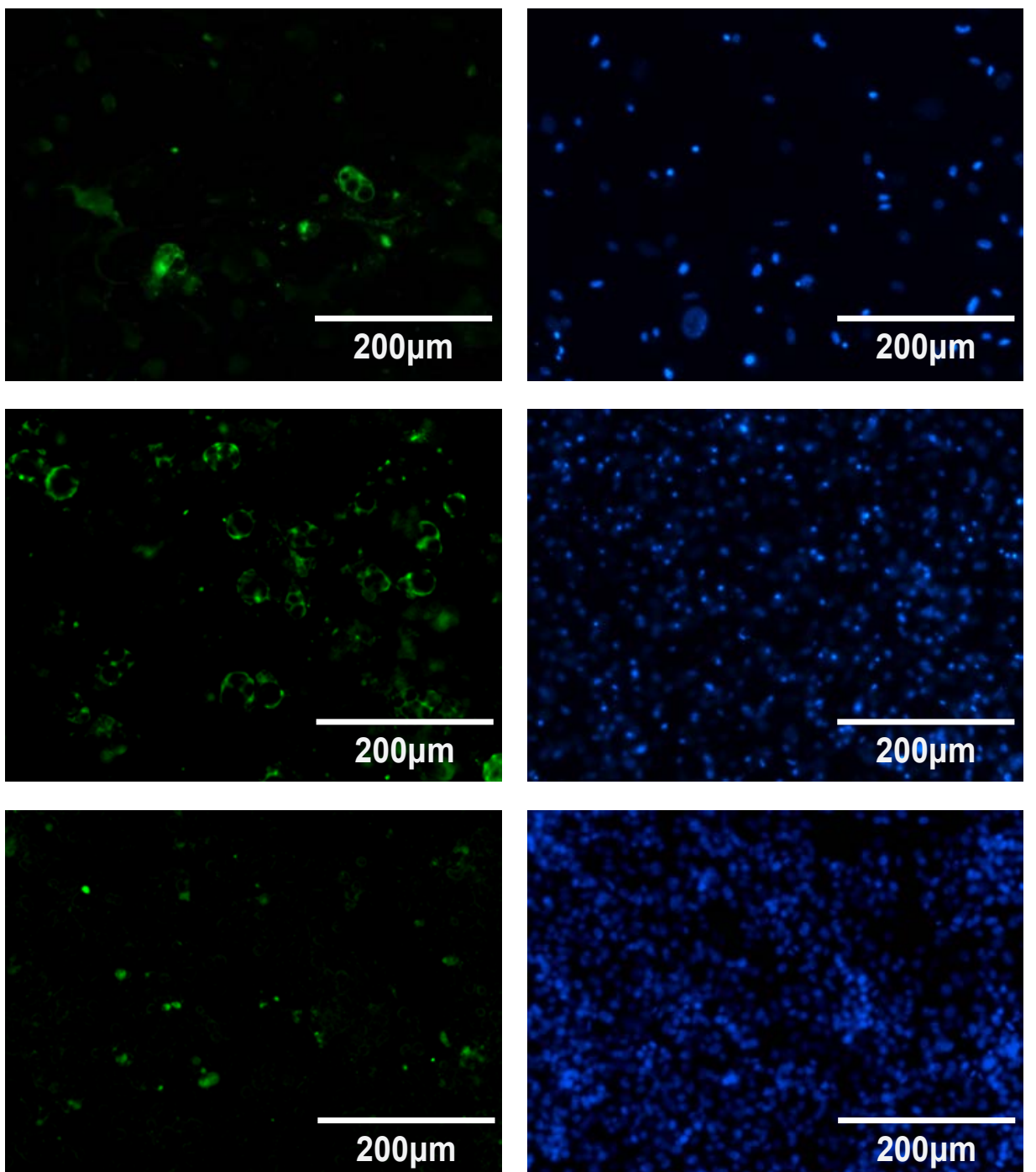

Merge
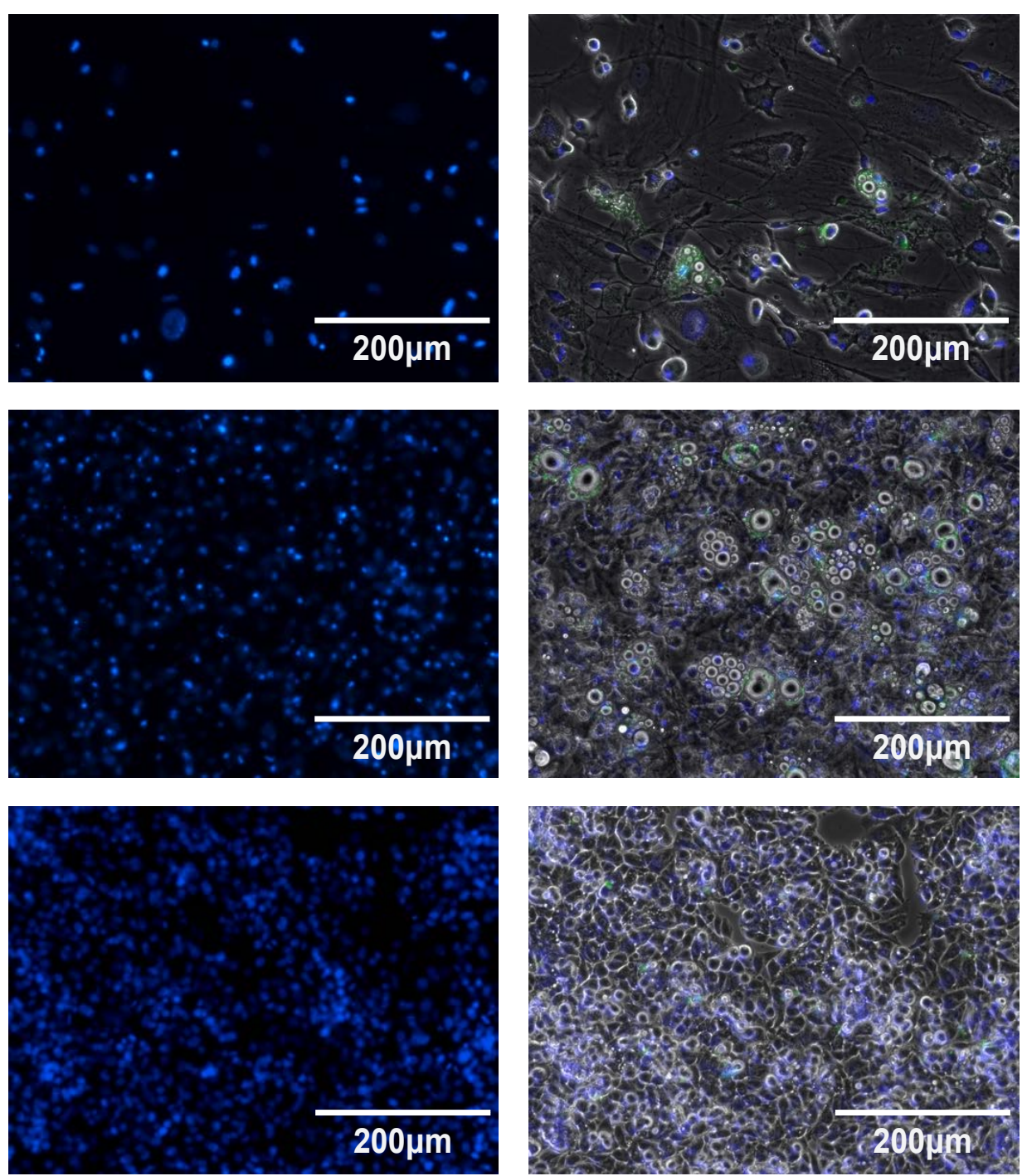

Figure 7 

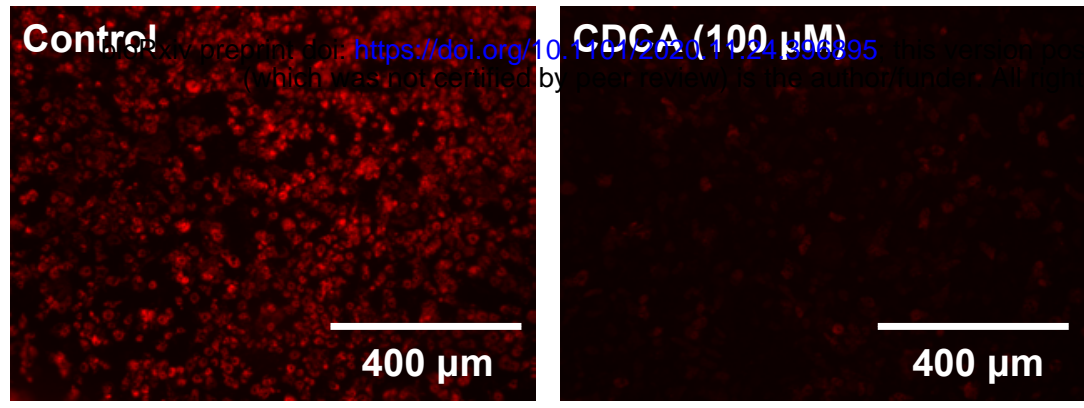
1.2

ted November 25 2020. The copyright holder for this preprint $s$ reserved. NoOrewse atleweet witheut germission.

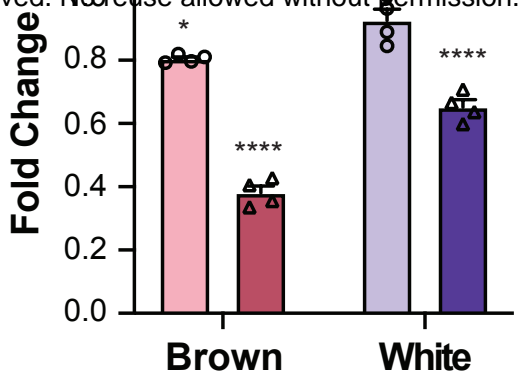

C

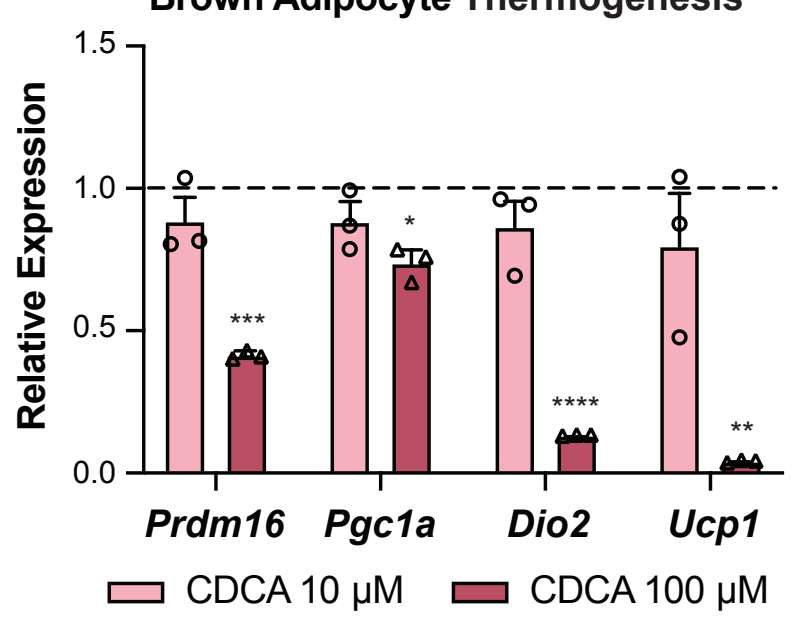

E

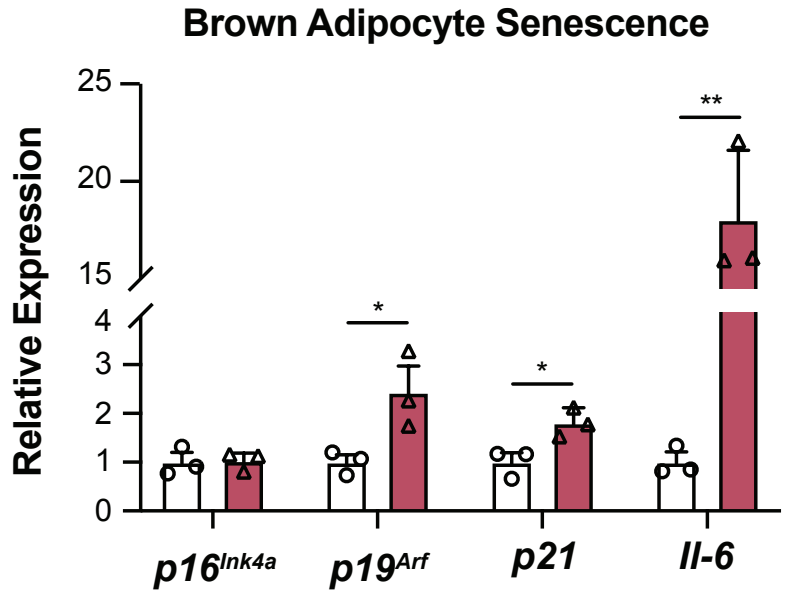

$\square$ Control $\square$ CDCA $100 \mu \mathrm{M}$
D White Adipocyte Mitochondrial Function

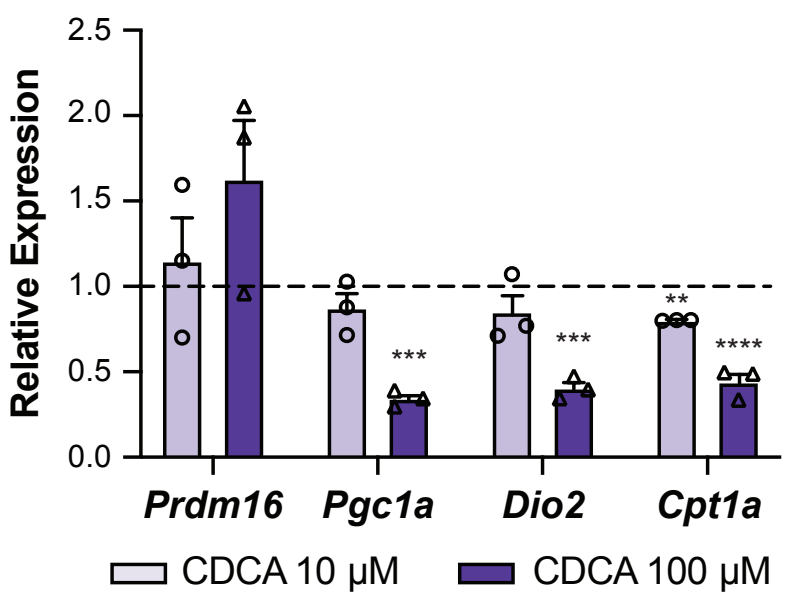

$\mathbf{F}$

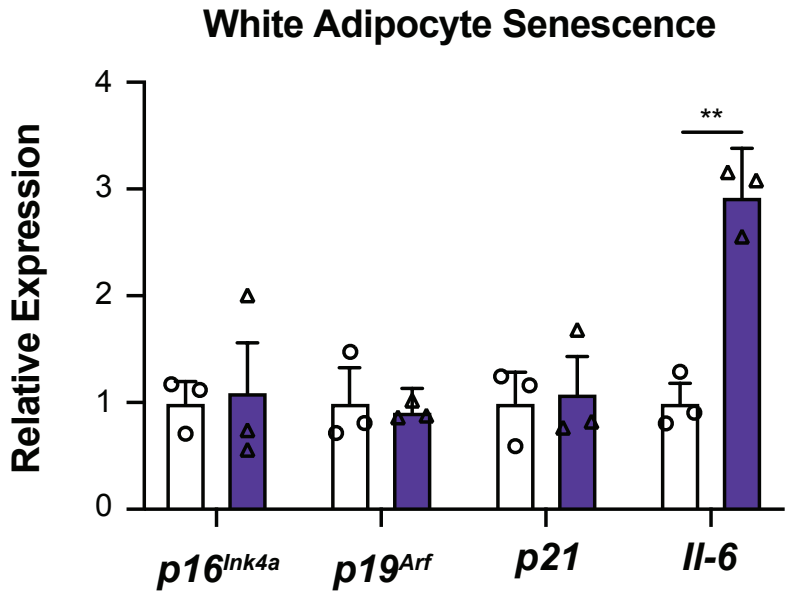

$\square$ Control $\square$ CDCA $100 \mu \mathrm{M}$

Figure 8 


\begin{tabular}{|c|c|c|c|c|c|c|c|}
\hline Unit: nM/mg & CA & TCA & GCA & CDCA & TCDCA & GCDCA & UDCA \\
\hline DKO-Liver & 0.002749 & 1.814166 & 0.003695 & 1.5323E-05 & 0.00253 & 2.6081E-05 & 2.2449E-05 \\
\hline \multirow[t]{2}{*}{ WT-Liver } & 0.000136 & 0.035177 & $<L O Q$ & 3.1553E-05 & 0.000764 & $<\mathrm{LOQ}$ & 3.34951E-05 \\
\hline & TUDCA & $\alpha-M C A$ & T- $\alpha-M C A$ & $\beta-M C A$ & T- $\beta$-MCA & DCA & \\
\hline DKO-Liver & 0.001092 & 0.000156 & 0.033728 & 0.02088491 & 0.127335 & 0.00011621 & \\
\hline \multirow[t]{2}{*}{ WT-Liver } & 0.001816 & 4.21E-05 & 0.001951 & 0.00085292 & 0.009911 & $<\mathrm{LOQ}$ & \\
\hline & TDCA & GDCA & TLCA & $\omega-M C A$ & T-w-MCA & GHDCA & \\
\hline DKO-Liver & 0.026629 & 1.44E-05 & 0.000101 & 0.0011469 & 0.037401 & $2.586 \mathrm{E}-05$ & \\
\hline WT-Liver & 0.004217 & $<\mathrm{LOQ}$ & 8.08E-05 & 9.9898E-05 & 0.00718 & $<\mathrm{LOQ}$ & \\
\hline
\end{tabular}

Table 1. Hepatic bile acid concentrations in DKO and WT mice 


\begin{tabular}{|c|c|c|c|c|c|c|c|c|}
\hline Unit: nM/mg & CA & TCA & GCA & CDCA & TCDCA & GCDCA & UDCA & TUDCA \\
\hline DKO-BAT-1 & 0.006076 & 0.043023 & 0.000147 & 1.29E-05 & 5.53E-05 & 2.56E-06 & 8.88E-05 & 0.000136 \\
\hline DKO-BAT-2 & 0.003566 & 0.00597 & 2.56E-06 & 3.02E-05 & 2.56E-06 & 1.18E-05 & 2.37E-05 & 2.56E-06 \\
\hline DKO-BAT-3 & 0.002302 & 0.006983 & 2.15E-05 & 2.97E-05 & 2.56E-06 & 2.56E-06 & 1.57E-05 & 2.24E-05 \\
\hline WT-BAT-1 & 0.000454 & 0.00017 & 2.56E-06 & $1.45 \mathrm{E}-05$ & $2.56 \mathrm{E}-06$ & 2.56E-06 & 2.56E-06 & $2.56 \mathrm{E}-06$ \\
\hline WT-BAT-2 & 0.000558 & 0.000191 & 2.56E-06 & 1.06E-05 & $2.56 \mathrm{E}-06$ & 2.56E-06 & 2.61E-05 & $2.56 \mathrm{E}-06$ \\
\hline WT-BAT-3 & 0.002769 & 0.000236 & 2.56E-06 & 2.56E-06 & 2.56E-06 & 2.56E-06 & 2.56E-06 & 2.56E-06 \\
\hline DKO-Gonadal WAT-1 & 0.000901 & 0.019745 & 6.78E-05 & 2.56E-06 & 4.94E-05 & 2.56E-06 & 2.14E-05 & 7.55E-05 \\
\hline DKO-Gonadal WAT-2 & 0.002032 & 0.001862 & $2.56 \mathrm{E}-06$ & 5.87E-05 & 2.56E-06 & 2.56E-06 & 4.49E-05 & 2.56E-06 \\
\hline DKO-Gonadal WAT-3 & 0.002705 & 0.002086 & $2.56 \mathrm{E}-06$ & 0.000057 & 2.56E-06 & 2.56E-06 & 0.000072 & $2.56 \mathrm{E}-06$ \\
\hline WT-Gonadal WAT-1 & 0.000361 & 0.000102 & 2.56E-06 & 7.98E-06 & 0 & 2.56E-06 & 2.56E-06 & 2.56E-06 \\
\hline WT-Gonadal WAT-2 & 0.002127 & 0.00071 & 2.56E-06 & $9.17 E-05$ & 2.56E-06 & $1.23 E-05$ & 3.01E-05 & 2.56E-06 \\
\hline \multirow[t]{2}{*}{ WT-Gonadal WAT-3 } & 0.001886 & 0.000152 & 2.56E-06 & 2.56E-06 & 2.56E-06 & 2.56E-06 & 2.56E-06 & 2.56E-06 \\
\hline & $\alpha-M C A$ & T- $\alpha-M C A$ & $\beta-M C A$ & T- $\beta$-MCA & DCA & TDCA & $\omega-M C A$ & T-w-MCA \\
\hline DKO-BAT-1 & $2.65 E-05$ & 0.002718 & 0.001613 & 0.003223 & 0.001776 & 0.000955 & 0.000293 & 0.001477 \\
\hline DKO-BAT-2 & 2.56E-06 & 0.000252 & 0.000204 & 0.000284 & 0.000274 & 0.000131 & 7.27E-05 & 0.000141 \\
\hline DKO-BAT-3 & 0 & 0.00013 & 0.000282 & 0.00049 & 0.000141 & 0.000358 & 2.56E-06 & 0.000143 \\
\hline WT-BAT-1 & 2.56E-06 & 0.000029 & 2.56E-06 & 7.52E-05 & 0.000142 & 2.24E-05 & 0 & 4.25E-05 \\
\hline WT-BAT-2 & 2.56E-06 & 2.74E-05 & $2.56 \mathrm{E}-06$ & 0.000062 & 8.66E-05 & 2.31E-05 & 2.56E-06 & $6.13 \mathrm{E}-05$ \\
\hline WT-BAT-3 & 2.56E-06 & 2.56E-06 & 2.56E-06 & 4.39E-05 & 5.07E-05 & 2.64E-05 & 2.56E-06 & 4.24E-05 \\
\hline DKO-Gonadal WAT-1 & 2.56E-06 & 0.001603 & 0.000987 & 0.002692 & 0.000619 & 0.000499 & 0.000235 & 0.000994 \\
\hline DKO-Gonadal WAT-2 & 2.56E-06 & 0.000111 & 4.46E-05 & 0.000148 & 9.64E-05 & 0.000057 & 2.56E-06 & 8.16E-05 \\
\hline DKO-Gonadal WAT-3 & 2.56E-06 & 6.94E-05 & 5.78E-05 & 0.000166 & 6.72E-05 & 4.56E-05 & 2.56E-06 & 4.95E-05 \\
\hline WT-Gonadal WAT-1 & 2.56E-06 & 2.56E-06 & 2.56E-06 & 3.43E-05 & 1.85E-05 & 2.56E-06 & 2.56E-06 & $1.95 E-05$ \\
\hline WT-Gonadal WAT-2 & 2.56E-06 & 0.000105 & 2.56E-06 & 0.000276 & 0.000429 & 5.24E-05 & 2.56E-06 & 0.00013 \\
\hline WT-Gonadal WAT-3 & 2.56E-06 & 2.74E-05 & 2.56E-06 & $9.47 E-05$ & 2.56E-05 & $2.56 \mathrm{E}-06$ & 0 & $6.14 \mathrm{E}-05$ \\
\hline
\end{tabular}

Table 2. Adipose bile acid concentrations in DKO and WT mice 


\begin{tabular}{|c|c|c|c|c|}
\hline Parameter & DKO & $\begin{array}{l}\text { CDCA-treated } \\
\text { adipocytes }\end{array}$ & Ucp1 KO & Aged \\
\hline Body weight & $\downarrow$ & & $\begin{array}{l}\text { NS (Enerback et al., 1997; Liu et al., } \\
\text { 2003) }\end{array}$ & $\begin{array}{l}\downarrow \text { (Seidell and Visscher, 2000) } \\
\text { NS (St-Onge and Gallagher, 2010) } \\
\uparrow \text { (Mancuso and Bouchard, 2019; Tchkonia } \\
\text { et al., 2010) }\end{array}$ \\
\hline WAT mass & $\downarrow$ & & $\begin{array}{l}\downarrow \text { (Enerback et al., 1997; Liu et al., } \\
2003)\end{array}$ & $\begin{array}{l}\downarrow \text { (Kirkland and Dobson, 1997; Kirkland et } \\
\text { al., 2002; Tchkonia et al., 2010) } \\
\uparrow(\text { St-Onge and Gallagher, 2010) }\end{array}$ \\
\hline BAT mass & $\downarrow$ & & $\begin{array}{l}\text { NS (Enerback et al., 1997; Liu et al., } \\
\text { 2003) }\end{array}$ & $\begin{array}{l}\downarrow \text { (Florez-Duquet and McDonald, 1998; } \\
\text { Zoico et al., 2019) }\end{array}$ \\
\hline $\begin{array}{l}\text { Mitochondrial } \\
\text { function }\end{array}$ & $\downarrow$ & $\downarrow$ & $\downarrow$ (Kazak et al., 2017) & $\begin{array}{l}\downarrow \text { (Florez-Duquet and McDonald, 1998; } \\
\text { Zoico et al., 2019) }\end{array}$ \\
\hline Ucp1 expression & $\downarrow$ & $\downarrow$ & $\begin{array}{l}\downarrow \text { (Enerback et al., 1997; Kazak et } \\
\text { al., 2017; Liu et al., 2003) }\end{array}$ & $\begin{array}{l}\downarrow \text { (Florez-Duquet and McDonald, 1998; } \\
\text { Zoico et al., 2019) }\end{array}$ \\
\hline Thermoregulation & $\downarrow$ & & $\begin{array}{l}\downarrow \text { (Enerback et al., 1997; Liu et al., } \\
2003 \text { ) }\end{array}$ & $\begin{array}{l}\downarrow \text { (Florez-Duquet and McDonald, 1998; } \\
\text { Zoico et al., 2019) }\end{array}$ \\
\hline
\end{tabular}

Table 3. Comparison summary between primary adipocyte cultures upon CDCA treatment, DKO, Ucp1 KO, and aged mice housed at room temperature 